TRANSACTIONS OF THE

AMERICAN MATHEMATICAL SOCIETY

Volume 290, Number 2, August 1985

\title{
ON A CLASS OF BANACH SPACES OF FUNCTIONS ASSOCIATED WITH THE NOTION OF ENTROPY
}

\author{
BY \\ BORIS KORENBLUM ${ }^{1}$
}

\begin{abstract}
A class of function spaces on the circle is introduced which contain all continuous functions of bounded variation but are included in the set of all continuous functions. The corresponding dual spaces consist of certain types of generalized measures. One application of these spaces is a new convergence test for Fourier series which includes both the Dirichlet-Jordan and the Dini-Lipschitz tests.
\end{abstract}

1. Introduction. Although it is hardly possible to overrate the importance and usefulness of such classical spaces of functions as $C, L^{p}, V$ and Lip, there are nevertheless many problems in analysis that fit badly into the pattern of these spaces. To give just one example, consider the problem of uniform convergence (or boundedness) of Fourier series for classes of bounded functions. The most relevant fact here is that the Dirichlet kernels $D_{n}(t)=\sin (n+1 / 2) t / \sin (t / 2)$ have unbounded $L^{1}$-norms or, equivalently, the $V$-norms of the indefinite integrals $\int D_{n}$ are unbounded, although their $C$-norms are bounded. Since we have few options other than to use the $C-V$ duality, we must impose an unnecessarily strong condition $f \in V$ to ensure that $\int_{\mathrm{T}} D_{n} f$ are bounded (the Dirichlet-Jordan test). That this condition $f \in V$ is indeed excessively strong is clearly shown by the Dini-Lipschitz test (see [1]): $\omega(\delta) \log \delta \rightarrow 0(\delta \rightarrow 0)$, where $\omega(\delta)$ is the modulus of continuity of $f$; this test guarantees the uniform convergence of the Fourier series of $f$. It is therefore reasonable to ask this question: Can the Dirichlet-Jordan test be generalized so that it might also include the Dini-Lipschitz test? A natural approach to this problem is to try to find an intermediate space $C^{\prime}$ between $C$ and $C \cap V$ (or between $L^{\infty}$ and $V$ - for the problem of boundedness), whose norm lies between the $C$-norm and the $V$-norm, and such that the $C^{\prime}$-norms of $\int D_{n}$ are bounded; at the same time the dual class of $C^{\prime}$ should include all Dini functions.

In fact, such a space $C^{\prime}$ exists; it is a special case in a class of spaces $C_{\kappa}$ with entropy norm. To explain this concept we first observe that the norm of the space (real) $C /\{$ constants $\}$, which is equal (up to the factor $1 / 2$ ) to

$$
\|x\|_{C}=\max _{t \in \mathbf{T}} x(t)-\min _{t \in \mathbf{T}} x(t)
$$

Received by the editors February 19, 1984 and, in revised form, November 1, 1984.

1980 Mathematics Subject Classification. Primary 46E15, 42A20.

${ }^{1}$ Supported by NSF grant MCS 82-01460. 
can be written as the integral

$$
\|x\|_{C}=\int_{-\infty}^{\infty} \kappa_{C}\left(E_{y}\right) d y
$$

where $E_{y}=\{t \in \mathbf{T}: x(t)=y\}(-\infty<y<\infty)$ are the level sets of $x(t)$ and $\kappa(E)$ is 0 or 1 depending on whether $E$ is empty or not. Similarly, the norm $\|x\|_{V}=\operatorname{Var} x(t)$ is equal to

$$
\|x\|_{V}=\int_{-\infty}^{\infty} \kappa_{V}\left(E_{y}\right) d y
$$

where $\kappa_{V}(E)=$ card $E$. These two cases $\kappa_{C}$ and $\kappa_{V}$ are the simplest examples of $\kappa$-entropy, and the only ones associated with known Banach spaces of functions.

DEFINITION 1. Let $\kappa(s)(0<s \leqslant 1)$ be a positive nondecreasing concave function such that $\kappa(1)=1$. The $\kappa$-entropy of a finite set $E \subset \mathbf{T}(E \neq \varnothing)$ is

$$
\kappa(E)=\sum_{j=1}^{n} \kappa\left(\left|I_{j}\right|\right),
$$

where $\left\{I_{j}\right\}_{1}^{n}$ are the complementary intervals of $E$ and $\left|I_{j}\right|$ their normalized lengths $(|\mathbf{T}|=1)$. For an infinite closed set $E \subset \mathbf{T}$ we define

$$
\kappa(E)=\sup \left\{\kappa\left(E_{1}\right): E_{1} \subset E \text { finite }\right\} ;
$$

we also set $\kappa(\varnothing)=0$.

DEFINITION 2. The $\kappa$-norm of a real continuous function $x(t)(t \in \mathbf{T})$ is

$$
\|x\|_{\kappa}=\int_{-\infty}^{\infty} \kappa\left(E_{y}\right) d y .
$$

The above restrictions on $\kappa(s)$ imply that

$$
s \leqslant \kappa(s) \leqslant 1 \quad(0<s \leqslant 1)
$$

with $\kappa(s)=s$ corresponding to $\|x\|_{C}$ and $\kappa(s)=1$ to $\|x\|_{V}$. We have $1 \leqslant \kappa(E) \leqslant$ card $E(E \neq \varnothing)$, and thus

$$
\|x\|_{C} \leqslant\|x\|_{\kappa} \leqslant\|x\|_{\nu} .
$$

Apart from $C$ and $V$, the following special cases of $\kappa$-entropy and $\kappa$-norm may be of particular interest:

(1) $\kappa(s)=s(|\log s|+1)$. In this case $\kappa(E)$ is called the Shannon entropy and $\|\cdot\|_{\kappa}$ the Shannon-entropy norm.

(2) $\kappa(s)=s^{\alpha}(0<\alpha<1) ; \kappa(E)$ is the Lipschitz entropy.

(3) $\kappa(s)=\left(1+\frac{1}{2}|\log s|\right)^{-1} ; \kappa(E)$ is the Dini entropy.

It is the Dini-entropy norm in combination with its dual $\kappa$-variation norm (see below) that yields our generalization of the Dirichlet-Jordan and the Dini-Lipschitz tests.

In this paper a general theory of $\kappa$-entropy and $\kappa$-variation spaces is presented. The main features of the theory are as follows:

(1) $C_{\kappa}$ is the space of continuous functions on $\mathbf{T}$ with finite $\kappa$-entropy norm (the real case is covered by Definition 2). It is by no means obvious that $C_{\kappa}$ is a Banach space; in fact, the proof of the triangle inequality is rather complicated. If $\kappa\left(0^{+}\right)=0$, 
$C_{\kappa}$ is a separable Banach space with the shift operator $T_{\tau} x=x(t-\tau)$ having the property $\left\|T_{\tau} x-x\right\|_{\kappa} \rightarrow 0(\tau \rightarrow 0)$ for all $x \in C_{\kappa}$.

(2) The linear functionals $F(x)$ on $C_{\kappa}$ (here and later we assume $\kappa\left(0^{+}\right)=0$ ) have the form

$$
F(x)=\int_{\mathbf{T}}^{\infty} x d \mu
$$

where $\mu$ is a premeasure of bounded $\kappa$-variation. The integral (1.6) is understood (in the real case) as follows:

$$
\int x d \mu=\int_{y_{0}}^{\infty} \mu\left(F_{y}\right) d y \quad\left(y_{0}=\min x(t)\right)
$$

where $F_{y}=\{t \in \mathbf{T}: x(t) \geqslant y\}$ are the Lebesgue sets of $x(t)$. For this definition of the integral to be consistent, the premeasure must be well defined on the sets $F_{y}$ for almost all $y$, which is the case if $\mu$ has bounded $\kappa$-variation and $x \in C_{\kappa}$. The norm $\|F\|$ of the functional (1.6) satisfies

$$
\|F\|=\frac{1}{2} \operatorname{Var}_{\kappa} \mu
$$

(see §5).

The notion of a premeasure and the definition of the $\kappa$-variation was first introduced in [2] for the case of the Shannon entropy in connection with an extension of the Nevanlinna theory (see also [3]).

(3) The Poisson integral of a real Borel measure is the difference of two positive harmonic functions in the unit disk (the Herglotz-Riesz theorem). An analogous result holds for premeasures, at least under some restrictions on the rate of growth of $\kappa(s) / s$ as $s \rightarrow 0$. The case of the Shannon entropy was considered in $[2,3]$.

(4) The space of premeasures of bounded $\kappa$-variation, denoted by $V$, contains a separable subspace $L_{\kappa}^{1}$ of $\kappa$-absolutely continuous premeasures. Premeasures belonging to $L_{\kappa}^{1}$ vanish on $\kappa$-Carleson sets. Every continuous function, whose modulus of continuity satisfies $\omega(\delta)=O(\kappa(\delta))(\delta \rightarrow 0)$, generates a premeasure of bounded $\kappa$-variation.

(5) The dual of $L_{\kappa}^{1}$ is $L_{\kappa}^{\infty}$, and $C_{\kappa}$ is the separable subspace of $L_{\kappa}^{\infty}$.

(6) With a slight adjustment of the $\kappa$-entropy norm, $C_{\kappa}$ and $L_{\kappa}^{\infty}$ become Banach algebras. Their analytic subalgebras $A_{\kappa}$ and $H_{\kappa}^{\infty}$ apparently share many properties with the classical algebras $A$ and $H^{\infty}$.

It should be clear from the above that the $\kappa$-entropy spaces, which span the gap between the uniform and the variation norm, together with their dual ( $\kappa$-variation) spaces, display a marked analogy with the classical spaces $C, V, L^{\infty}, A, H^{1}, H^{\infty}$. There is, however, one important difference: there seems to be no place in our theory for anything like the $L^{p}$ and $H^{p}$ spaces $(1<p<\infty)$.

There is good reason to believe that both the analogy and the difference may help us better understand some recent discoveries in complex and harmonic analysis.

The author wishes to thank R. Dabrowski and R. O'Neil for valuable discussions and suggestions. The author also expresses his gratitude to the referee who detected numerous imperfections in the original manuscript. 


\section{Preliminaries.}

2.1. (a) $\mathbf{T}=\mathbf{R} / 2 \pi \mathbf{Z}$ is the additive group of real numbers modulo $2 \pi$.

(b) $\partial \mathbf{D}=\{\zeta \in \mathbf{C}:|\zeta|=1\}$ is the isomorphic multiplicative group (the unit circle).

(c) $\mathbf{D}=\{z \in \mathbf{C}:|z|<1\}$ is the open unit disk.

(d) $|F|$ denotes the normalized Lebesgue measure of a set $F \subset \mathbf{T}$ or $F \subset \partial \mathbf{D}$, so that $|\mathbf{T}|=|\partial \mathbf{D}|=1$.

(e) $\operatorname{dist}\left(t_{1}, t_{2}\right)=(1 / 2 \pi) \min \left\{\left|t_{1}-t_{2}-2 n \pi\right|: n \in \mathbf{Z}\right\}, \quad\left(t_{1}, t_{2} \in \mathbf{T}\right) ; \operatorname{dist}\left(\zeta_{1}, \zeta_{2}\right)$ $\left(\zeta_{1}, \zeta_{2} \in \partial \mathbf{D}\right)$ is the (normalized) length of the shorter arc between $\zeta_{1}$ and $\zeta_{2}$; $\operatorname{dist}(t, F)=\inf \{\operatorname{dist}(t, \tau): \tau \in F\}$.

2.2. $\mathscr{R} L^{\infty}\left(L^{\infty}\right)$ is the space of real (complex) essentially bounded measurable functions $x=x(t)(t \in \mathbf{T})$ with the ess sup-norm. Given an $x \in \mathscr{R} L^{\infty}$, we define

(a) $\bar{\mu}(t)=\bar{\mu}(t ; x)=\lim _{\delta \rightarrow 0} \operatorname{ess} \sup \{x(\tau): t-\delta<\tau<t+\delta\}$.

(b) $\underline{\mu}(t)=\underline{\mu}(t ; x)=\lim _{\delta \rightarrow 0} \operatorname{ess} \inf \{x(\tau): t-\delta<\tau<t+\delta\}$.

(c) $\bar{\omega}(t)=\bar{\omega}(t ; x)=\bar{\mu}(t)-\mu(t)$ (essential oscillation at $t)$.

(d) $\Gamma[x]=\{(t, y) \in \mathbf{T} \times \mathbf{R}: \underline{\mu}(t) \leqslant y \leqslant \bar{\mu}(t)\}$ (the graph of $x(t)$ ).

(e) $E_{y}=E_{y}[x]=\{t \in \mathrm{T}:(t, y) \in \Gamma[x]\}(-\infty<y<\infty)$.

(f) $F_{y}=F_{y}[x]=\bigcup_{\eta \geqslant y} E_{\eta}[x]$.

The $E_{y}$ 's are called the level sets and the $F_{y}$ 's are the Lebesgue sets of $x(t)$. The following properties are easily proved:

(i)

$$
\bar{\mu}(t)=\limsup _{\tau \rightarrow t} \bar{\mu}(\tau), \quad \underline{\mu}(t)=\liminf _{\tau-t} \underline{\mu}(\tau) .
$$

(ii) $\Gamma[x]$ is a closed connected subset of $\mathbf{T} \times \mathbf{R}$.

(iii) $E_{y}, F_{y}$ and $\Omega_{\delta}=\{t \in \mathbf{T}: \omega(t) \geqslant \delta\}$ are closed subsets of $\mathbf{T}$ for every $y \in \mathbf{R}$, $\delta \geqslant 0\left(\Omega_{0}=\mathbf{T}\right)$.

The discontinuity set $\Omega=\Omega[x]=\cup_{\delta>0} \Omega_{\delta}$ is thus an $F_{\sigma}$-set. If $\Omega$ does not contain intervals of positive length, then it is of the first Baire category. If $|\Omega|=0$, then $x(t)$ is Riemann integrable. ${ }^{2}$

$\mathscr{R} C$ is the subspace of $\mathscr{R} L^{\infty}$ consisting of continuous functions $x=x(t)$. For an $x \in \mathscr{R} C$ we have $\Gamma(x)=\{(t, y) \in \mathbf{T} \times \mathbf{R}: y=x(t)\}, E_{y}=\{t \in \mathbf{T}: x(t)=y\}, F_{y}$ $=\{t \in \mathbf{T}: x(t) \geqslant y\}$ and

$$
x(t)=y_{0}+\int_{y_{0}}^{\infty} \chi_{y}(t) d y,
$$

where $y_{0}=\min \{x(t): t \in \mathbf{T}\}$ and $\chi_{y}(t)=\chi\left(t ; F_{y}\right)$ is the characteristic function of $F_{y}[x]$. (2.2.1) remains valid for any $x \in \mathscr{R} L^{\infty}$, provided that $t \notin \Omega[x]$. If $x(t)$ is Riemann integrable, then (2.2.1) holds almost everywhere.

2.3. $\kappa(s)$ will always denote a positive nondecreasing concave function in $(0 ; 1]$ such that $\kappa(1)=1$. These conditions imply that $\kappa(s)$ is continuous, $\kappa^{\prime}(x)$ exists a.e. and is nonincreasing, and $s \leqslant \kappa(s) \leqslant 1$. We shall assume that $\kappa^{\prime}(s)$ is defined everywhere by putting $\kappa^{\prime}(s)=\kappa^{\prime}\left(s^{-}\right)$.

\footnotetext{
${ }^{2}$ More precisely, equivalent to a Riemann integrable function.
} 
The $\kappa$-entropy of a finite set $E \subset \mathbf{T}$ is

$$
\kappa(E)=\sum_{j=1}^{n} \kappa\left(\left|I_{j}\right|\right),
$$

where $\left\{I_{j}\right\}_{1}^{n}$ are the complementary intervals of $E ; \kappa(\varnothing)=0$. For an infinite closed set $E$ we define

$$
\kappa(E)=\sup \left\{\kappa\left(E_{1}\right): E_{1} \subset E \text { finite }\right\} .
$$

The following properties are easily deduced from the definition.

(i) $\kappa(E)=1$ if card $E=1$.

(ii) If $\kappa(s) \neq s$ and card $E>1$, then $\kappa(E)>1$.

(iii) If $\kappa(s)=s$, then $\kappa(E)=1$ for any $E \neq \varnothing$.

(iv) If $\kappa(s)=1$, then $\kappa(E)=$ card $E$.

(v) $\kappa\left(E_{1} \cup E_{2}\right) \leqslant \kappa\left(E_{1}\right)+\kappa\left(E_{2}\right)$.

(vi) $\kappa(E+t)=\kappa(E)(t \in \mathbf{T})$.

(vii) If $\kappa\left(0^{+}\right)=0$, then the $\kappa$-entropy of an arbitrary closed set $E \subset \mathbf{T}$ can be expressed in the form of an integral:

$$
\kappa(E)=\int_{\mathbf{T}} \kappa^{\prime}(2 \operatorname{dist}(t, E)) d t
$$

where $d t$ is the normalized Lebesgue measure and $\kappa^{\prime}(0)=\lim _{s \rightarrow 0}(\kappa(s) / s) ; \kappa^{\prime}(0)=\infty$ is not excluded.

(viii) If $\kappa^{\prime}(0)=\infty$, then $\kappa(F)<\infty$ implies $|F|=\infty$.

(ix) If card $E=n$, then $\kappa(F \leqslant n \kappa(1 / n))$, the equality holding for $\left|I_{1}\right|=\left|I_{2}\right|=\cdots$ $=\left|I_{n}\right|=1 / n$.

2.4. The $\kappa$-norm of an $x \in \mathscr{R} L^{\infty}$ is

$$
\|x\|_{\kappa}=\int_{-\infty}^{\infty} \kappa\left(E_{y}[x]\right) d y .
$$

The following two extreme cases are classical.

(a) $\kappa(s)=s$. Here we have

$$
\|x\|_{\kappa}=\operatorname{ess} \sup x(t)-\operatorname{ess} \inf x(t)
$$

thus the $\kappa$-norm is equivalent to the norm of $x$ in $L^{\infty} /\{$ constants $\}$.

(b) $x(s)=1$. In this case

$$
\|x\|_{\kappa}=\int_{-\infty}^{\infty}\left(\operatorname{card} E_{y}\right) d y=\operatorname{Var} x(t) .
$$

If $\kappa^{\prime}(0)<\infty$, the $\kappa$-norm is equivalent to the norm of the space $L^{\infty} /$ \{constants\}; if $\kappa\left(0^{+}\right)>0$, the $\kappa$-norm is equivalent to $\operatorname{Var} x$. We shall exclude these two cases and assume from now on that $\kappa(s)$ satisfies:

(a) $\lim _{s \rightarrow 0} \kappa(s)=0$;

(b) $\kappa^{\prime}(0)=\lim _{s \rightarrow 0}(x(s) / s)=\infty$.

It follows from the above that $\kappa(E)<\infty$ implies $|E|=0$. Since $1 \leqslant \kappa(E) \leqslant$ card $E$ $(E \neq \varnothing)$, we have

$$
\text { ess sup } x(t)-\operatorname{essinf} x(t) \leqslant\|x\|_{\kappa} \leqslant \operatorname{Var} x(t) .
$$


3. The Banach spaces $L_{\kappa}^{\infty}, C_{\kappa}$.

3.1. TheOREM. For arbitrary $x, x_{1}, x_{2} \in \mathscr{R} L^{\infty}$ and $\lambda \in \mathbf{R}$

$$
\begin{aligned}
\left\|x_{1}+x_{2}\right\|_{\kappa} & \leqslant\left\|x_{1}\right\|_{\kappa}+\left\|x_{2}\right\|_{\kappa}, \\
\|\lambda x\|_{\kappa} & =|\lambda|\|x\|_{\kappa} .
\end{aligned}
$$

Whereas (3.1.2) follows directly from (2.4.1), the proof of (3.1.1) is more complicated. We shall prove it in three steps: first for piecewise constant functions, then for real continuous functions, and finally for arbitrary real $L^{\infty}$-functions.

3.2. A set $F \subset \mathbf{T}$ will be called simple if it is the union of a finite number of disjoint closed intervals. The class of all simple sets $F$ will be denoted by $\mathscr{F} ; \varnothing$ and $\mathbf{T}$ are included in $\mathscr{F}$.

Lemma 1. Let $F_{1}, F_{2} \in \mathscr{F}$ and let $\chi_{1}(t)=\chi\left(t ; F_{1}\right)$ and $\chi_{2}=\chi\left(t ; F_{2}\right)$ be the characteristic functions of $F_{1}$ and $F_{2}$. Then

$$
\left\|\chi_{1}+\chi_{2}\right\|_{\kappa} \leqslant\left\|\chi_{1}\right\|_{\kappa}+\left\|\chi_{2}\right\|_{\kappa} \text {. }
$$

Proof. $^{3}(3.2 .1)$ is equivalent to

$$
\kappa\left(\partial\left(F_{1} \cup F_{2}\right)\right)+\kappa\left(\partial\left(F_{1} \cap F_{2}\right)\right) \leqslant \kappa\left(\partial F_{1}\right)+\kappa\left(\partial F_{2}\right) .
$$

Without loss of generality we can assume that $\partial F_{1}$ and $\partial F_{2}$ are disjoint. Consider the following four functions:

$$
\begin{aligned}
& h_{1}(t)=\kappa^{\prime}\left(2 \operatorname{dist}\left(t, \partial\left(F_{1} \cup F_{2}\right)\right)\right), \\
& h_{2}(t)=\kappa^{\prime}\left(2 \operatorname{dist}\left(t, \partial\left(F_{1} \cap F_{2}\right)\right)\right), \\
& h_{3}(t)=\kappa^{\prime}\left(2 \operatorname{dist}\left(t, \partial F_{1}\right)\right), \\
& h_{4}(t)=\kappa^{\prime}\left(2 \operatorname{dist}\left(t, \partial F_{2}\right)\right) .
\end{aligned}
$$

By (2.3.3), in order to prove (3.2.2) it is enough to show that

$$
h_{1}(t)+h_{2}(t) \leqslant h_{3}(t)+h_{4}(t) \quad(t \in \mathbf{T}) .
$$

Let $t_{0} \in \mathbf{T}$ be arbitrary and let $\operatorname{dist}\left(t_{0}, \partial F_{1} \cup \partial F_{2}\right)=d_{0}=\operatorname{dist}\left(t_{0}, t_{1}\right)$, where $t_{1} \in$ $\partial F_{1} \cup \partial F_{2}$; let, for instance, $t_{1} \in \partial F_{1}$. There are two possibilities:

(a) $t_{1} \in I$, where $I$ is one of the components of $F_{2}$;

(b) $t_{1} \in I^{\prime}$, where $I^{\prime}$ is one of the components of $\mathbf{T} \backslash F_{2}$.

In case (a) we have $t_{1} \in \partial\left(F_{1} \cap F_{2}\right)$ and $\left(t_{0}-d_{0}, t_{0}+d_{0}\right) \subset I \subset F_{1} \cup F_{2}$; therefore $\operatorname{dist}\left(t_{0}, \partial\left(F_{1} \cap F_{2}\right)\right)=d_{0}=d\left(t_{0}, \partial F_{1}\right)$ and $\operatorname{dist}\left(t_{0}, \partial\left(F_{1} \cup F_{2}\right)\right) \geqslant \operatorname{dist}\left(t_{0}, \partial F_{2}\right)$. Since $\kappa^{\prime}(s)$ is nonincreasing, we get $h_{2}\left(t_{0}\right)=\kappa^{\prime}\left(2 d_{0}\right)=h_{3}\left(t_{0}\right)$ and $h_{1}\left(t_{0}\right) \leqslant h_{4}\left(t_{0}\right)$. Similarly, in case (b) we obtain $t_{1} \in \partial\left(F_{1} \cup F_{2}\right)$ and $\left(t_{0}-d_{0}, t_{0}+d_{0}\right) \subset I^{\prime} \subset$ $\mathbf{T} \backslash\left(F_{1} \cap F_{2}\right)$; thus $h_{1}\left(t_{0}\right)=\kappa^{\prime}\left(2 d_{0}\right)=h_{3}\left(t_{0}\right)$ and $h_{2}\left(t_{0}\right) \leqslant h_{4}\left(t_{0}\right)$. In both cases (3.2.3) holds at $t=t_{0}$, which proves our lemma.

REMARK. If $F_{1} \supseteq F_{2},(3.2 .1)$ becomes an equality.

\footnotetext{
${ }^{3}$ This proof is based on an idea suggested to the author by Romuald Dabrowski; the original proof by the author was more complicated.
} 
LeMma 2. Let $F_{1}, F_{2}, \ldots, F_{n} \in \mathscr{F}$ and let $\chi_{j}(t)=\chi\left(t ; F_{j}\right)$ be the characteristic function of $F_{j}(j=1,2, \ldots, n)$. Then

$$
\left\|x_{1}+x_{2}+\cdots+\chi_{n}\right\|_{\kappa} \leqslant\left\|x_{1}\right\|_{\kappa}+\left\|x_{2}\right\|_{\kappa}+\cdots+\left\|\chi_{n}\right\|_{\kappa} .
$$

Proof. Suppose (3.2.4) holds for some $n \geqslant 2$ and arbitrary $F_{1}, F_{2}, \ldots, F_{n}$. We have $x(t)=\chi_{1}(t)+\chi_{2}(t)+\cdots+\chi_{n}(t)=\chi\left(t ; F_{1}^{\prime}\right)+\chi\left(t ; F_{2}^{\prime}\right)+\cdots+\chi_{k}\left(t ; F_{k}^{\prime}\right)$, where $F_{1}^{\prime} \supseteq F_{2}^{\prime} \supseteq \cdots \supseteq F_{k}^{\prime}(1 \leqslant k \leqslant n)$ are the Lebesgue sets of $x(t): F_{j}^{\prime}=\{t$; $x(t) \geqslant j\}$. By our assumption, (3.2.4) holds, i.e.

$$
\|x\|_{\kappa}=\kappa\left(\partial F_{1}^{\prime}\right)+\kappa\left(\partial F_{2}^{\prime}\right)+\cdots+\kappa\left(\partial F_{k}^{\prime}\right) \leqslant \kappa\left(\partial F_{1}\right)+\kappa\left(\partial F_{2}\right)+\cdots+\kappa\left(\partial F_{n}\right) .
$$

If we add $\chi_{n+1}(t)=\chi\left(t ; F_{n+1}\right)$, we get

$$
\chi(t)+\chi_{n+1}(t)=\chi\left(t ; F_{1}^{\prime} \cup F_{n+1}\right)+\chi\left(t ; F_{1}^{\prime} \cap F_{n+1}\right)+\sum_{j=2}^{k} \chi\left(t ; F_{j}^{\prime}\right) .
$$

Now, by our assumption and by (3.2.2)

$$
\begin{aligned}
\left\|x+\chi_{n+1}\right\|_{\kappa} & =\kappa\left(\partial\left(F_{1}^{\prime} \cup F_{n+1}\right)\right)+\left\|\chi\left(t ; F_{1}^{\prime} \cap F_{n+1}\right)+\sum_{j=2}^{k} \chi\left(t ; F_{j}^{\prime}\right)\right\|_{\kappa} \\
& \leqslant \kappa\left(\partial\left(F_{1}^{\prime} \cup F_{n+1}\right)\right)+\kappa\left(\partial\left(F_{1}^{\prime} \cap F_{n+1}\right)\right)+\sum_{j=2}^{k} \kappa\left(\partial F_{j}^{\prime}\right) \\
& \leqslant \kappa\left(\partial F_{1}^{\prime}\right)+\kappa\left(\partial F_{n+1}\right)+\sum_{j=2}^{k} \kappa\left(\partial F_{j}^{\prime}\right) \leqslant \sum_{j=1}^{n+1} \kappa\left(\partial F_{j}\right) .
\end{aligned}
$$

LEMMA 3. (3.1.1) holds for functions $x_{1}, x_{2}$ of the form

$$
x_{j}(t)=\sum_{\nu=1}^{n_{j}} \chi\left(t ; F_{\nu}^{(j)}\right) \quad\left(F_{\nu}^{(j)} \in \mathscr{F} ; j=1,2\right) .
$$

Proof. Since the Lebesgue sets of $x_{j}$ belong to the class $\mathscr{F}$, we may assume that $F^{(j)}$ are already the Lebesgue sets of $x_{j}(j=1,2)$ i.e. $F_{1}^{(j)} \supseteq F_{2}^{(j)} \supseteq \cdots \supseteq F_{n}^{(j)}$ $(j=1,2)$. Now,

$$
\left\|x_{j}\right\|_{\kappa}=\sum_{\nu=1}^{n_{j}} \kappa\left(\partial F_{\nu}^{(j)}\right) \quad(j=1,2)
$$

and, by Lemma 2 ,

$$
\left\|x_{1}+x_{2}\right\|_{\kappa} \leqslant \sum_{\nu=1}^{n_{1}} \kappa\left(\partial F_{\nu}^{(1)}\right)+\sum_{\nu=1}^{n_{2}} \kappa\left(\partial F_{\nu}^{(2)}\right)=\left\|x_{1}\right\|_{\kappa}+\left\|x_{2}\right\|_{\kappa} .
$$

LEMMA 4. (3.1.1) holds for piecewise constant functions, i.e. for functions of the form

$$
x(t)=\sum_{j=1}^{n} C_{j} \chi\left(t ; F_{j}\right) \quad\left(C_{j} \in \mathbf{R}, F_{j} \in \mathscr{F}\right) .
$$


Proof. By Lemma 3, this is true for integer $C_{j}$; by the homogeneity of the $\kappa$-norm, this is also true for rational $C_{j}$; finally, by an obvious approximation argument, (3.1.1) holds for all functions of the form (3.2.5).

3.3. The next step is to establish (3.1.1) for real continuous functions; this requires a certain process of approximation in the $\kappa$-norm of a continuous function by step functions.

Lemma 1. Suppose that $x \in \mathscr{R} C,\|x\|_{\kappa}<\infty$. For any $\varepsilon>0$ there is a function $x_{\varepsilon}(t)$ of the form (3.2.5) such that

$$
\left\|x-x_{\varepsilon}\right\|_{\kappa}<\varepsilon
$$

Proof. Without loss of generality we can assume that $\min \{x(t): t=\mathbf{T}\}=0$. Choose some $h, 0<h<\max x(t)$, and construct the system of lines in $\mathbf{T} \times \mathbf{R}$ : $l_{k}=\{(t, k h): t \in \mathbf{T}\}, k \in \mathbf{Z}$. Let $S_{k}$ be the open strip $\{(t, y): t \in \mathbf{T},(k-1) h<y$ $<(k+1) h\}$ of width $2 h$ containing $l_{k}$. Construct a finite system of disjoint open intervals $I_{j}=\left\{t \in \mathbf{T}: t_{j}<t<t_{j}^{\prime}\right\}(j=1,2, \ldots, n), U_{j} \bar{I}_{j}=\mathbf{T}$, and a function $k(j)$ $\in \mathbf{Z}$ such that

(i) $x\left(t_{j}\right)=k(j) h$;

(ii) $\left\{(t, x(t)): t \in I_{j}\right\} \subset S_{k(j)}$;

(iii) $\left|x\left(t_{j}^{\prime}\right)-x\left(t_{j}\right)\right|=h$.

We now set

$$
x^{h}(t)=k(j) h \quad\left(t \in I_{j} ; j=1,2, \ldots, n\right),
$$

and we shall prove that

$$
\lim _{h \rightarrow 0}\left\|x^{h}-x\right\|_{\kappa}=0
$$

By (2.3.3) and (2.4.1)

$$
\|x\|_{\kappa}=\int_{\mathbf{T} \times \mathbf{R}} \kappa^{\prime}\left(2 \operatorname{dist}\left(t, E_{y}[x]\right)\right) d t d y<\infty .
$$

Construct the rectangles $R_{j}=\left\{(t, y) \in \mathbf{T} \times \mathbf{R}: t \in \bar{I}_{j}, y \in \bar{S}_{k(j)}\right\}$ and let $R^{h}=$ $\bigcup_{j=1}^{n} R_{j}$. Since the (two-dimensional) measure of $R^{h}$ is $2 h,(3.3 .4)$ implies

$$
\lim _{h \rightarrow 0} \int_{R^{h}} \kappa^{\prime}\left(2 \operatorname{dist}\left(t, E_{y}[x]\right)\right) d t d y=0 .
$$

For every $j$ the set $E_{y}[x] \cap I_{j}$ is nonempty either for $k(j) h<y<(k(j)+1) h$ or for $k(j) h>y>(k(j)-1) h$, and for such $y$

$$
\int_{I_{j}} \kappa^{\prime}\left(2 \operatorname{dist}\left(t, E_{y}[x]\right)\right) d t \geqslant \frac{1}{2} \kappa\left(2\left|I_{j}\right|\right) .
$$

Thus

$$
\lim _{h \rightarrow 0} h \sum_{j=1}^{n} \kappa\left(\left|I_{j}\right|\right)=0 .
$$


The graph of $r(t)=x(t)-x^{h}(t)$ lies in the strip $|y| \leqslant h$. The section of $\Gamma[r]$ corresponding to $\bar{I}_{j}$ is obtained from the corresponding section of $\Gamma[x]$ (lying in $R_{j}$ ) by shifting it vertically by $-k(j) h$ and adding to it parts of vertical lines through $t_{j}$, $t_{j}^{\prime}$; therefore

$$
\begin{aligned}
\|r\|_{\kappa} & =\int_{\mathbf{T} \times(-h, h)} \kappa^{\prime}\left(2 \operatorname{dist}\left(t, E_{y}[r]\right)\right) d t d y \\
& \leqslant \int_{R^{h}} \kappa^{\prime}\left(2 \operatorname{dist}\left(t, E_{y}[x]\right)\right) d t d y+2 h \sum_{j=1}^{n} \kappa\left(\left|I_{j}\right|\right) \rightarrow 0 \quad(h \rightarrow 0)
\end{aligned}
$$

by (3.3.5) and (3.3.6), which proves (3.3.3).

LEMMA 2. The function $x^{h}(t)$ satisfies

$$
\lim _{h \rightarrow 0}\left\|x^{h}\right\|=\|x\|_{\kappa}
$$

Proof. It suffices to prove this for an arbitrary sequence $h_{n} \downarrow 0$. Suppose that $y$ does not belong to the countable set $\left\{k h_{n} ; k=0,1,2, \ldots ; n=1,2, \ldots\right\}$ and to the (at most countable) set of local extreme values of $x(t)$. A complementary interval $I=(a, b)$ of $E_{y}[x]$ contains at most one point of each $E_{y}\left[x^{h_{n}}\right]$; moreover, for an arbitrary $\varepsilon>0$ and sufficiently large $n E_{y}\left[x^{h_{n}}\right] \cap(a+\varepsilon, b-\varepsilon)=\varnothing$, but $E_{y}\left[x^{h_{n}}\right]$ $\cap(a-\varepsilon, a+\varepsilon) \neq \varnothing$ and $E_{y}\left[x^{h_{n}}\right] \cap(b-\varepsilon, b+\varepsilon) \neq \varnothing$. Therefore

$$
\begin{aligned}
2 \kappa(|I|) & =2 \int_{I} \kappa^{\prime}\left(2 \operatorname{dist}\left(t, E_{y}[x]\right)\right) d t \\
& \geqslant \int_{I} \kappa^{\prime}\left(2 \operatorname{dist}\left(t, E_{y}\left[x^{h_{n}}\right]\right)\right) d t \rightarrow \kappa(|I|)
\end{aligned}
$$

as $n \rightarrow \infty$. Taking the sum over all complementary intervals $I$ we find

$$
2 \kappa\left(E_{y}[x]\right) \geqslant \kappa\left(E_{y}\left[x^{h_{n}}\right]\right) \rightarrow \kappa\left(E_{y}[x]\right) .
$$

Integrating now with respect to $y$ we obtain (3.3.7) by dominated convergence theorem.

LEMMA 3. (3.1.1) holds for arbitrary $x_{1}, x_{2} \in \mathscr{R} C$.

Proof. Choose two sequences of step functions of the form (3.2.5), $\left\{x_{1}^{(n)}\right\}$ and $\left\{x_{2}^{(n)}\right\}$, such that

$$
\lim _{n \rightarrow \infty}\left\|x_{j}^{(n)}-x_{j}\right\|_{\kappa}=0, \quad \lim _{n \rightarrow \infty}\left\|x_{j}^{(n)}\right\|_{\kappa}=\left\|x_{j}\right\|_{\kappa} \quad(j=1,2) .
$$

By Lemma 4 in $\S 3.2,\left\|x_{1}^{(n)}+x_{2}^{(n)}\right\|_{\kappa} \leqslant\left\|x_{1}^{(n)}\right\|_{\kappa}+\left\|x_{2}^{(n)}\right\|_{\kappa}$. Since $x_{1}^{(n)}(t)+x_{2}^{(n)}(t) \rightarrow$ $x_{1}(t)+x_{2}(t)$ uniformly on $\mathrm{T}$, we have

$$
\liminf _{n \rightarrow \infty} \kappa\left(E_{y}\left[x_{1}^{(n)}+x_{2}^{(n)}\right]\right) \geqslant \kappa\left(E_{y}\left[x_{1}+x_{2}\right]\right)
$$

for all $y$ except the (at most countable) set of extreme values of $x_{1}(t)+x_{2}(t)$. By Fatou's lemma

$$
\liminf _{n \rightarrow \infty} \int_{-\infty}^{\infty} \kappa\left(E_{y}\left[x_{1}^{(n)}+x_{2}^{(n)}\right]\right) d y \geqslant \int_{-\infty}^{\infty} \kappa\left(E_{y}\left[x_{1}+x_{2}\right]\right) d y
$$


or, equivalently, liminf $\left\|x_{1}^{(n)}+x_{2}^{(n)}\right\|_{\kappa} \geqslant\left\|x_{1}+x_{2}\right\|_{\kappa}$. Using (3.3.10) and Lemma 4 of \$3.2, we obtain $\left\|x_{1}+x_{2}\right\|_{\kappa} \leqslant\left\|x_{1}\right\|_{\kappa}+\left\|x_{2}\right\|_{\kappa}$.

REMARK 1. In the above proof we used only the second formula (3.3.10), which can be established independently of Lemma 1 . However, Lemma 1 will be needed later to prove the continuity of the shift operator in the $\kappa$-norm.

REMARK 2. In any normed space (3.3.7) is, of course, implied by (3.3.3); however, this argument cannot be used for proving (3.3.7), since we have not yet established the general triangle inequality.

3.4. We proceed now to proving (3.1.1) for arbitrary $x_{1}, x_{2} \in \mathscr{R} L^{\infty}$.

Lemma 1. If $x \in \mathscr{R} L^{\infty}$ and $\|x\|_{\kappa}<\infty$, then $|\Omega[x]|=0$, i.e. $x(t)$ is Riemann integrable. $^{4}$

Proof. Formula (3.3.4), which holds for any $x \in \mathscr{R} L^{\infty}$ with $\|x\|_{\kappa}<\infty$, shows that the set $G_{\delta}=\left\{(t, y) \in \mathbf{T} \times \mathbf{R}:(t, y) \in \Gamma[x], t \in \Omega_{\delta}\right\}$ is of (two-dimensional) Lebesgue measure zero for every $\delta>0$, since the integrand in (3.3.4) is equal to $+\infty$ on that set. On the other hand, $\left|G_{\delta}\right| \geqslant \delta\left|\Omega_{\delta}\right|$, which implies $\left|\Omega_{\delta}\right|=0$ and $|\Omega|=0$.

Let $x \in \mathscr{R} L^{\infty}$ be Riemann integrable, and let $F_{1} \subset F_{2} \subset \cdots$ be a sequence of closed subsets of $\mathbf{T}$ such that

(i) $\bigcup_{j=1}^{\infty} F_{j} \subset \mathbf{T} \backslash \Omega[x]$;

(ii) $\left|\mathbf{T} \backslash \bigcup_{j=1}^{\infty} F_{j}\right|=0$.

Since $|\Omega[x]|=0$, such a sequence $\left\{F_{j}\right\}$ exists. We construct now a sequence of continuous functions $\left\{x_{n}(t)\right\}_{1}^{\infty}: x_{n}(t)=x(t)\left(t \in F_{n}\right), x_{n}(t)$ is linear on each component of $\mathbf{T} \backslash F_{n}$.

LEMMA 2. The above sequence $\left\{x_{n}(t)\right\}$ satisfies:

(i) $\kappa\left(E_{y}\left[x_{n}\right]\right) \leqslant 3 \kappa\left(E_{y}[x]\right)(y \in \mathbf{R} ; n=1,2, \ldots)$.

(ii) $\lim _{n \rightarrow \infty} \kappa\left(E_{y}\left[x_{n}\right]\right)=\kappa\left(E_{y}[x]\right)$ a.e.

(iii) $\lim _{n \rightarrow \infty}\left\|x_{n}\right\|_{\kappa}=\|x\|_{\kappa}$.

Proof. (i) follows from the observation that each complementary interval of $E_{y}[x]$ ( $y$ fixed) contains at most two points of $E_{y}\left[x_{n}\right]$.

(ii) Using essentially the same argument as in the proof of Lemma 2 in $\S 3.3$, we see that (ii) holds everywhere except a finite or countable set of values of $y$ corresponding to local maxima and minima of the graph $\Gamma[x]$.

(iii) By dominated convergence,

$$
\lim _{n \rightarrow \infty}\left\|x_{n}\right\|_{\kappa}=\lim _{n \rightarrow \infty} \int_{-\infty}^{\infty} \kappa\left(E_{y}\left[x_{n}\right]\right) d y=\int_{-\infty}^{\infty} \kappa\left(E_{y}[x]\right) d y=\|x\|_{\kappa} .
$$

We can now complete the proof of (3.1.1). Assume $\left\|x_{1}\right\|_{\kappa}<\infty$ and $\left\|x_{2}\right\|_{\kappa}<\infty$. Choose a sequence of closed sets $F_{1} \subset F_{2} \subset \cdots$ such that

$$
\bigcup_{n>0} F_{n} \subset \mathbf{T} \backslash\left(\Omega\left[x_{1}\right] \cup \Omega\left[x_{2}\right]\right), \quad\left|\mathbf{T} \backslash \bigcup_{n>0} F_{n}\right|=0
$$

\footnotetext{
${ }^{4}$ See footnote 2 .
} 
and construct (as described above) two sequences $\left\{x_{1}^{(n)}(t)\right\}$ and $\left\{x_{2}^{(n)}(t)\right\}$ corresponding to $x_{1}(t)$ and $x_{2}(t)$ respectively. By the construction, we have $x_{1}^{(n)}(t)+$ $x_{2}^{(n)}(t)=x_{1}(t)+x_{2}(t)\left(t \in F_{n}\right) ; x_{1}^{(n)}(t)+x_{2}^{(n)}(t)$ is linear on complementary intervals of $F_{n}$. Since (3.1.1) holds for continuous functions, and using Lemma 2, we obtain

$$
\begin{aligned}
\left\|x_{1}+x_{2}\right\|_{\kappa} & =\lim _{n \rightarrow \infty}\left\|x_{1}^{(n)}+x_{2}^{(n)}\right\|_{\kappa} \leqslant \lim _{n \rightarrow \infty}\left\|x_{1}^{(n)}\right\|_{\kappa}+\lim _{n \rightarrow \infty}\left\|x_{2}^{(n)}\right\|_{\kappa} \\
& =\left\|x_{1}\right\|_{\kappa}+\left\|x_{2}\right\|_{\kappa} .
\end{aligned}
$$

Thus our theorem has been proved completely.

3.5. The linear space of functions $x \in \mathscr{R} L^{\infty}$ such that $\|x\|_{\kappa}<\infty$ will be denoted by $\mathscr{R} L_{\kappa}^{\infty}$; two functions $x_{1}, x_{2} \in \mathscr{R} L_{\kappa}^{\infty}$ such that $x_{1}(t)-x_{2}(t)=$ const are considered identical as elements of $\mathscr{R} L_{\kappa}^{\infty}$.

THEOREM. $\mathscr{R} L_{\kappa}^{\infty}$ is a Banach space.

PROOF. In view of Theorem 3.1, we need only to prove the completeness of $\mathscr{R} L_{\kappa}^{\infty}$. Let $\left\{x_{n}\right\}$ be a Cauchy sequence, i.e.

$$
\lim _{m, n \rightarrow \infty}\left\|x_{m}-x_{n}\right\|_{\kappa}=0 .
$$

By (2.4.2) $x_{n}$ converges uniformly (i.e. in the norm of $L^{\infty} /$ constants $\}$ ) to some element $x \in \mathscr{R} L^{\infty}$. We want to prove that $x \in \mathscr{R} L_{\kappa}^{\infty}$ and

$$
\lim _{n \rightarrow \infty}\left\|x-x_{n}\right\|_{\kappa}=0 \text {. }
$$

Given an $\varepsilon>0$, let $N=N_{\varepsilon}$ be such that

$$
\left\|x_{m}-x_{n}\right\|_{\kappa}<\varepsilon \quad(m, n>N) .
$$

For a fixed $n>N$ and $m \rightarrow \infty$ we have $x_{m}(t)-x_{n}(t) \rightarrow x(t)-x_{n}(t)$ uniformly. To complete the proof we can use the following

LEMMA. If $f_{n} \in \mathscr{R} L_{\kappa}^{\infty},\left\|f_{n}\right\|_{\kappa} \leqslant A(n=1,2, \ldots)$ and $f_{n} \rightarrow f(n \rightarrow \infty)$ uniformly, then $\|f\|_{\kappa} \leqslant A$.

Proof of THE Lemma. If $y$ does not belong to the (at most countable) set of essential extreme values of $f(t)$, then for every complementary interval $I$ of $E_{y}[f]$ there is a sequence $\left\{I_{n}\right\}$ of complementáry intervals of $E_{y}\left[f_{n}\right]$ such that the endpoints of $I_{n}$ tend to the corresponding endpoints of $I$ and therefore $\kappa\left(\left|I_{n}\right|\right) \rightarrow$ $\kappa(|I|)$. This implies that

$$
\liminf _{n \rightarrow \infty} \kappa\left(E_{y}\left[f_{n}\right]\right) \geqslant \kappa\left(E_{y}[f]\right),
$$

and therefore by Fatou's lemma

$$
\|f\|_{\kappa}=\int_{-\infty}^{\infty} \kappa\left(E_{y}[f]\right) d y \leqslant \liminf _{n \rightarrow \infty} \int_{-\infty}^{\infty} \kappa\left(E_{y}\left[f_{n}\right]\right) d y \leqslant A .
$$

The Lemma and the Theorem are proved.

3.6. The subspace of $\mathscr{R} L_{\kappa}^{\infty}$ consisting of continuous functions will be denoted by $\mathscr{R} C_{\kappa}$. 
THEOREM. An element $x \in \mathscr{R} L_{\kappa}^{\infty}$ belongs to $\mathscr{R} C_{\kappa}$ if and only if

$$
\lim _{\tau \rightarrow 0}\left\|x_{\tau}-x\right\|_{\kappa}=0
$$

where $x_{\tau}(t)=x(t-\tau)$.

Proof. The "if" part is trivial. Suppose now that $x \in \mathscr{R} C_{\kappa}$, and let $x^{h}$ be the step function (3.3.2). By (3.3.3) and (3.3.6), for an arbitrary $\varepsilon>0$ and sufficiently small $h$

$$
\left\|x^{h}-x\right\|_{\kappa}<\varepsilon, \quad h \sum_{j=1}^{h} \kappa\left(\left|I_{j}\right|\right)<\varepsilon,
$$

where $\left\{I_{j}\right\}_{1}^{n}$ are the complementary intervals of the discontinuity set $\Omega_{h}$ of $x^{h}$. For sufficiently small $\tau$, say $|\tau|<\delta$, the function $x^{h}(t)-x^{h}(t-\tau)$ takes on only three values, $-h, 0, h$, and the set of its discontinuities will be close to $\Omega_{h}$. This implies that there is some $\delta>0$ such that for $|\tau|<\delta$

$$
\left\|x^{h}-x_{\tau}^{h}\right\|_{\kappa} \leqslant 4 h \sum_{1}^{n} \kappa\left(\left|I_{j}\right|\right)<4 \varepsilon .
$$

Combining (3.6.2) and (3.6.3) we get

$$
\left\|x_{\tau}-x\right\|_{\kappa} \leqslant\left\|x^{h}-x\right\|_{\kappa}+\left\|x_{\tau}^{h}-x^{h}\right\|_{\kappa}+\left\|\left(x-x^{h}\right)_{\tau}\right\|_{\kappa} \leqslant 6 \varepsilon
$$

which proves the Theorem.

Corollary. $\mathscr{R} C_{\kappa}$ is a separable Banach space: trigonometric polynomials are dense in $\mathscr{R} C_{\kappa}$.

3.7. The complex space $L_{\kappa}^{\infty}$ consists of functions $z(t)=x(t)+i y(t)$, where $x, y \in \mathscr{R} L_{\kappa}^{\infty}$; to ensure the homogeneity of the norm we have to define it as follows:

$$
\|z\|_{\kappa}=\max \left\{\left(\left\|\lambda_{1} x-\lambda_{2} y\right\|_{\kappa}^{2}+\left\|\lambda_{2} x+\lambda_{1} y\right\|_{\kappa}^{2}\right)^{1 / 2}: \lambda_{1}^{2}+\lambda_{2}^{2}=1\right\} \text {. }
$$

As is easily seen,

$$
\sqrt{\|x\|_{\kappa}^{2}+\|y\|_{\kappa}^{2}} \leqslant\|z\|_{\kappa} \leqslant\|x\|_{\kappa}+\|y\|_{\kappa} .
$$

$C_{\kappa}$ is the subspace of $L_{\kappa}^{\infty}$ consisting of continuous functions. By the above, $C_{\kappa}$ is a separable Banach space.

\section{Premeasures and the $\kappa$-integral.}

4.1. Some adaptation of the classical notions of Lebesgue and Stieltjes integrals will be needed to obtain a complete description of linear functionals in the spaces $C_{\kappa}$. First we introduce the notion of a premeasure.

Definition 1. $\mathscr{I}$ is the set of all open, closed and half-closed intervals $I \subset \mathbf{T}$,

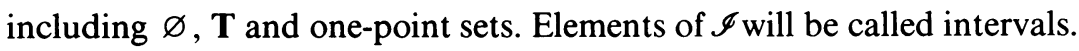

Definition 2. A function $\mu: \mathscr{I} \rightarrow \mathbf{C}$ is called a premeasure if the following conditions hold:

(i) $\mu(\varnothing)=\mu(\mathbf{T})=0$;

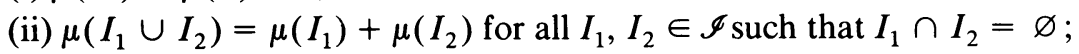

(iii) If $I_{1} \supset I_{2} \supset \cdots$ is a decreasing sequence of intervals and $\cap_{n=1}^{\infty} I_{n}=\varnothing$, then $\lim _{n \rightarrow \infty} \mu\left(I_{n}\right)=0$. 
These conditions imply that the function $\tilde{\mu}(t)=\mu((0, t])(0<t \leqslant 2 \pi)$ is continuous to the right, has left-hand limit values $\tilde{\mu}\left(t^{-}\right)=\mu((0, t))$, and $\tilde{\mu}\left(0^{+}\right)=\tilde{\mu}(2 \pi)=0$.

DEFINITION 3. The $\kappa$-variation of a premeasure $\mu$ is

$$
\operatorname{Var}_{\kappa} \mu=\sup \frac{\sum_{1}^{n}\left|\mu\left(I_{j}\right)\right|}{\sum_{1}^{n} \kappa\left(\left|I_{j}\right|\right)},
$$

where supremum is taken over all finite systems of disjoint intervals $I_{j}$ such that $\bigcup_{j} I_{j}=\mathbf{T}$. If $\operatorname{Var}_{\kappa} \mu<\infty, \mu$ will be called a premeasure of bounded $\kappa$-variation and the corresponding function $\tilde{\mu}(t)$ will be called a function of bounded $\kappa$-variation.

The (linear) space of premeasures of bounded $\kappa$-variation will be denoted by $V_{\kappa}$; equipped with the norm $\|\mu\|=\operatorname{Var}_{\kappa} \mu$, it becomes a Banach space. $\mathscr{R} V_{\kappa}$ will denote the (real) Banach space of real premeasures of bounded $\kappa$-variation.

Definition 4. For an open set $G \subset \mathbf{T}$ such that $\kappa(\partial G)<\infty$, and for a $\mu \in V_{\kappa}$, we set

$$
\mu(G)=\sum_{j} \mu\left(I_{j}\right)
$$

where $\left\{I_{j}\right\}$ are the components of $G$. For a closed set $F \subset \mathbf{T}$ with $\kappa(\partial F)<\infty$ we define

$$
\mu(F)=-\mu(\mathbf{T} \backslash F) .
$$

The series (4.1.2) is absolutely convergent because of $\kappa(\partial G)<\infty$. Thus, a premeasure of bounded $\kappa$-variation extends naturally to all open and closed sets whose boundary has a finite $\kappa$-entropy.

Definition 5. A closed set $E \subset \mathbf{T}$ with $\kappa(E) \leqslant \infty$ is called a $\kappa$-Carleson set. Every $\kappa$-Carleson set is of Lebesgue measure 0 (see $\$ 2.3$ ).

From (4.1.1), (4.1.2) and (4.1.3) we derive that $(G=\mathbf{T} \backslash F)$

$$
\begin{aligned}
|\mu(F)| & =|\mu(G)|=\frac{1}{2}(|\mu(F)|+|\mu(G)|) \\
& \leqslant \frac{1}{2}\left(\operatorname{Var}_{\kappa} \mu\right) \kappa(\partial F)=\frac{1}{2}\left(\operatorname{Var}_{\kappa} \mu\right) \kappa(\partial G) .
\end{aligned}
$$

4.2. We proceed now to introducing the notion of a $\kappa$-integral; it will enable us to integrate functions $x \in C_{\kappa}$ with respect to premeasures of bounded $\kappa$-variation. Heuristically, if $x \in \mathscr{R} C_{\kappa}$ and $\mu \in V_{\kappa}$, we can use (2.2.1) and formally invert the order of integrations:

$$
\int x d \mu=\int\left(y_{0}+\int_{y_{0}}^{\infty} \chi\left(t ; F_{y}\right) d y\right) d \mu=\int_{-\infty}^{\infty} \mu\left(F_{y}[x]\right) d y .
$$

This provides the basis for the following

Definition. For an $x \in \mathscr{R} C_{\kappa}$ and $\mu \in V_{\kappa}$ the $\kappa$-integral is defined by the formula

$$
\int x d \mu=\int_{-\infty}^{\infty} \mu\left(F_{y}[x]\right) d y,
$$

where the latter integral exists as a Lebesgue integral, due to (4.1.4) and (2.4.1). If $x=x_{1}+i x_{2} \in C_{\kappa}\left(x_{1}, x_{2} \in \mathscr{R} C_{\kappa}\right)$, then

$$
\int x d \mu=\int x_{1} d \mu+i \int x_{2} d \mu \text {. }
$$


Proposition. The $\kappa$-integral (4.2.2) is bilinear in $x \in C_{\kappa}$ and $\mu \in V_{\kappa}$. Moreover,

$$
\left|\int x d \mu\right| \leqslant \frac{1}{2}\|x\|_{\kappa}\left(\operatorname{Var}_{\kappa} \mu\right) \quad\left(x \in \mathscr{R} C_{\kappa}, \mu \in V_{\kappa}\right) \text {. }
$$

Proof. Observe now that definition (4.2.1) is still consistent if $x(t)$ is piecewise continuous, provided that the discontinuity sets of $x(t)$ and $\tilde{\mu}(t)$ are disjoint. An elementary argument then shows that

$$
\int\left(C_{1} x_{1}+C_{2} x_{2}\right) d \mu=C_{1} \int x_{1} d \mu+C_{2} \int x_{2} d \mu
$$

if at least one of the functions $x_{1}, x_{2}$ is of the form (3.2.5). By an approximation argument involving Lemma 1 in $\S 3.3$, this immediately extends to arbitrary $x_{1}, x_{2} \in$ $\mathscr{R} C_{\kappa}$.

For complex-valued functions $z(t) \in C$ we obtain, using (3.7.1):

$$
\left|\int z d \mu\right| \leqslant \frac{\sqrt{2}}{2}\|z\|_{\kappa}\left(\operatorname{Var}_{\kappa} \mu\right) \text {. }
$$

5. $V_{\kappa}$ as the dual of $C_{\kappa}$.

5.1. TheOREM. (a) Every (real) linear functional $F$ on $\mathscr{R C}$ has the form of a $\kappa$-integral:

$$
F(x)=\int x d \mu
$$

where $\mu \in \mathscr{R} V_{\kappa}$, and its norm $\|F\|^{\mathscr{R}}$ satisfies

$$
\|F\|^{\mathscr{R}}=\frac{1}{2} \operatorname{Var}_{\kappa} \mu \text {. }
$$

(b) Every linear functional $F$ on $C_{\kappa}$ has the form (5.1.1), where $\mu \in V_{\kappa}$, and its norm satisfies

$$
\frac{1}{2 \sqrt{2}} \operatorname{Var}_{\kappa} \mu \leqslant\|F\| \leqslant \frac{\sqrt{2}}{2} \operatorname{Var}_{\kappa} \mu
$$

Proof. (a) Observe first that every premeasure $\mu \in \mathscr{R} V_{\kappa}$ generates by (5.1.1) a linear functional $F$ on $\mathscr{R} C_{\kappa}$ whose norm is $\leqslant \frac{1}{2} \operatorname{Var}_{\kappa} \mu$ by (4.2.3). To express $\mu$ or, equivalently, the function $\tilde{\mu}(t)=\mu((0, t])$, in terms of $F$, consider the sequence $\left\{\varphi_{t, n}(s)\right\}_{n=1}^{\infty}$ of continuous functions

$$
\varphi_{t, n}(s)=\left\{\begin{array}{l}
1, \quad 2^{-n} \leqslant s \leqslant t \\
0, \quad t \notin\left(0, t+2^{-n}\right) \\
\text { linear on }\left[0,2^{-n}\right] \text { and }\left[t, t+2^{-n}\right]
\end{array}\right.
$$

Clearly,

$$
\tilde{\mu}(t)=\lim _{n \rightarrow \infty} F\left(\varphi_{t, n}\right) .
$$

(Of course, this formula presumes that the functional $F$ is a priori known to have the form (5.1.1).) Now, we want to show that the limit in (5.1.4) exists for an arbitrary 
real linear functional $\mathscr{F}$ on $\mathscr{R} C_{\kappa}$. Assuming the contrary, we can pick a subsequence $\left\{\varphi_{t, n_{k}}\right\}$ such that

$$
\left|F\left(\varphi_{t, n_{k}}\right)-F\left(\varphi_{t, n_{k+1}}\right)\right|>a>0 \quad(k=1,2, \ldots) .
$$

The function $\psi_{k}(s)=\varphi_{t, n_{k}}(s)-\varphi_{t, n_{k+1}}(s)$ vanishes outside a set $\left(0, \delta_{k}\right) \cup(t, t+$ $\left.\delta_{k}\right)$, where $\delta_{k} \rightarrow 0(k \rightarrow \infty)$. We construct now a new sequence of functions $\left\{\tilde{\psi}_{k}(s)\right\}$ as follows: we start with $\psi_{1}$, shift it to the right so slightly as not to upset (5.1.5), and thus obtain $\tilde{\psi}_{1}(s)=\psi_{1}\left(s-\tilde{\delta}_{1}\right)$. Then we pick a $\psi_{k_{2}}, k_{2}>1$, whose support has an empty intersection with that of $\tilde{\psi}_{1}$, shift it slightly (say by $\tilde{\delta}_{2}$ ) to the right and put $\tilde{\psi}_{2}=\psi_{k_{2}}\left(s-\tilde{\delta}_{2}\right)$. We then continue in the same way, and thus arrive at a sequence $\left\{\tilde{\psi}_{k}\right\}$. It is easily seen that by choosing $\tilde{\delta}_{k}$ at each step sufficiently small we can ensure that the constructed sequence $\left\{\tilde{\psi}_{k}\right\}$ has the following properties:

(i) $\tilde{\psi}_{k}(s)$ vanishes outside the set $\left(\delta_{k}^{\prime}, \delta_{k}^{\prime \prime}\right) \cup\left(t+\delta_{k}^{\prime}, t+\delta_{k}^{\prime \prime}\right)$, where $\left[\delta_{k}^{\prime}, \delta_{k}^{\prime \prime}\right]$, $\left[t+\delta_{k}^{\prime}, t+\delta_{k}^{\prime \prime}\right](k=1,2, \ldots)$ are disjoint intervals, and $\delta_{k}^{\prime} \rightarrow 0, \delta_{k}^{\prime \prime} \rightarrow 0$;

(ii) the set $\left\{\delta_{1}^{\prime \prime}, \delta_{1}^{\prime}, \delta_{2}^{\prime \prime}, \delta_{2}^{\prime}, \ldots, 0\right\} \cup\left\{t+\delta_{1}^{\prime \prime}, t+\delta_{1}^{\prime}, t+\delta_{2}^{\prime \prime}, t+\delta_{2}^{\prime}, \ldots, t\right\}$ is a $\kappa$ Carleson set;

(iii) the graph of $\tilde{\psi}_{k}(s)$ on each of the intervals $\left[\delta_{k}^{\prime}, \delta_{k}^{\prime \prime}\right],\left[t+\delta_{k}^{\prime}, t+\delta_{k}^{\prime \prime}\right](k=$ $1,2, \ldots)$ consists of two linear parts;

(iv) $\left|\tilde{\psi}_{k}(s)\right| \leqslant 1(s \in \mathbf{T} ; k=1,2, \ldots)$;

(v) $\left|F\left(\tilde{\psi}_{k}\right)\right|>a>0(k=1,2, \ldots)$.

Consider now

$$
x(s)=\sum_{k=1}^{\infty}\left(\operatorname{sgn} F\left(\tilde{\psi}_{k}\right)\right) \tilde{\psi}_{k}(s) / k .
$$

Using (i)-(v) we can easily prove that $x \in \mathscr{R} C_{\kappa}$; however,

$$
F(x)=\sum_{k=1}^{\infty}\left(\operatorname{sgn} F\left(\tilde{\psi}_{k}\right)\right) F\left(\tilde{\psi}_{k}\right) / k=\sum_{k=1}^{\infty}\left|F\left(\tilde{\psi}_{k}\right)\right| / k \geqslant a \sum_{k=1}^{\infty} 1 / k=\infty .
$$

This contradiction proves that the limit in (5.1.4) exists for every linear functional $F$ on $\mathscr{R} C_{\kappa}$; it also proves that there is a "natural" norm-preserving extension of the functional $F$ to all real piecewise constant functions given by the formula (5.1.1), where $\mu$ is the premeasure corresponding to the function (5.1.4). The premeasure $\mu$ is clearly of bounded $\kappa$-variation, because the functional $F$ is bounded. Since every $x \in \mathscr{R} C_{\kappa}$ can be approximated in the $\kappa$-norm by piecewise constant functions, (5.1.1) holds for all $x \in \mathscr{R} C_{\kappa}$.

To prove (5.1.2), observe first that (4.2.3) implies

$$
\|F\|^{\mathscr{P}} \leqslant \frac{1}{2} \operatorname{Var}_{\kappa} \mu .
$$

From the definition of the $k$-variation it follows that for every $\varepsilon>0$ there is some $t_{1}<t_{2}<\cdots<t_{n}<t_{n+1}=t_{1}+2 \pi$ such that

$$
\sum_{j=1}^{n}\left|\tilde{\mu}\left(t_{j+1}\right)-\tilde{\mu}\left(t_{j}\right)\right| \geqslant\left(\operatorname{Var}_{\kappa} \mu-\varepsilon\right) \sum_{j=1}^{n} \kappa\left(\frac{1}{2 \pi}\left(t_{j+1}-t_{j}\right)\right) .
$$


Now put

$$
x(t)=\operatorname{sgn}\left(\tilde{\mu}\left(t_{j+1}\right)-\tilde{\mu}\left(t_{j}\right)\right) \quad\left(t_{j}<t \leqslant t_{j+1} ; j=1,2, \ldots, n\right) .
$$

We have

$$
F(x) \geqslant\left(\operatorname{Var}_{\kappa} \mu-\varepsilon\right) \sum_{j=1}^{n} \kappa\left(\frac{1}{2 \pi}\left(t_{j+1}-t_{j}\right)\right) .
$$

On the other hand,

$$
\|x\|_{\kappa} \leqslant 2 \sum_{j=1}^{n} \kappa\left(\frac{1}{2 \pi}\left(t_{j+1}-t_{j}\right)\right)
$$

and therefore

$$
F(x) \geqslant \frac{1}{2}\left(\operatorname{Var}_{\kappa} \mu-\varepsilon\right)\|x\|_{\kappa} .
$$

Since our "natural" extension of the functional $F$ to the piecewise constant $x(t)$ is norm-preserving, we get $\|F\|^{R} \geqslant \frac{1}{2} \operatorname{Var}_{\kappa} \mu$, which together with (5.1.6) proves (5.1.2).

(b) follows directly from (a) and (3.7.1).

5.2. Definition. A real premeasure $\mu$ is called $\kappa$-bounded (above) if there is a constant $C_{\mu}>0$ such that

$$
\mu(I) \leqslant C_{\mu} \kappa(|I|) \quad(\forall I \in \mathscr{I}) .
$$

Clearly, a $\kappa$-bounded premeasure is of bounded $\kappa$-variation. The following theorem is analogous to the classical result about representation of a Borel measure as the difference of two positive measures.

THEOREM. Let $\mu$ be a real premeasure of bounded $\kappa$-variation. Then $\mu=\mu_{1}-\mu_{2}$, where $\mu_{1}$ and $\mu_{2}$ are some $\kappa$-bounded premeasures; moreover, they can be chosen so that their constants $C_{\mu_{1}}$ and $C_{\mu_{2}}$ (see (5.2.1)) do not exceed $\operatorname{Var}_{\kappa} \mu$.

Proof. For the case of the Shannon entropy the proof was given in [2, p. 206]. In the general case the proof differs only in minor details.

5.3. Every premeasure $\mu$ generates a harmonic function $u(z)$ in $\mathbf{D}$ by the formula

$$
u(z)^{2}=\int \frac{1-|z|^{2}}{\left|e^{i t}-z\right|^{2}} d \mu(t)
$$

$u(z)$ will be called the Poisson integral of $d \mu$. If $\mu$ is a real Borel measure, then $u(z)$ is the difference of two positive harmonic functions, and vice versa (Herglotz-Riesz). A similar result holds for premeasures, at least if $\kappa^{\prime}(s)$ is slowly increasing as $s \rightarrow 0$.

Definition. $\kappa(s)$ is said to be of $\mathrm{S}$ type ( $\mathrm{S}$ for Shannon) if there is a constant $C>0$ such that

$$
\kappa^{\prime}\left(s^{2}\right) \leqslant C \kappa^{\prime}(s) \quad(0<s<1) .
$$

In the case of the Shannon entropy $\kappa(s)=s(|\log s|+1)$ it was shown in [2] that the Poisson integral $u$ of a $\kappa$-bounded premeasure $\mu$ satisfies

$$
u(z) \leqslant \gamma C_{\mu} \log (1-|z|) \quad\left(\frac{1}{2}<|z|<1\right),
$$


where $C_{\mu}$ is the constant in (5.2.1) and $\gamma$ is an absolute constant; conversely, every real harmonic function $u$ in D satisfying $u(0)=0$ and (5.3.3) is the Poisson integral of a $\kappa$-bounded premeasure. This result extends to all $\kappa(s)$ of $\mathrm{S}$ type, as shown by the following theorem whose proof will be given elsewhere.

THEOREM. There is a 1-1 correspondence between the class of $\kappa$-bounded premeasures and the class $h_{\kappa}^{+}$of real harmonic functions in $\mathbf{D}$ satisfying $u(0)=0$ and

$$
u(z) \leqslant C_{u} \kappa^{\prime}(1-|z|) \quad\left(\frac{1}{2} \leqslant|z|<1\right),
$$

where $\kappa(s)$ is a given function of $\mathrm{S}$ type. In one direction this correspondence is given by the formula (5.3.1) and in the other by

$$
\frac{1}{2}(\mu(I)+\mu(\bar{I}))=\lim _{r \rightarrow 1^{-}} \int_{I} u\left(r e^{i t}\right) d t
$$

where $I \subset \partial \mathbf{D}$ is an arbitrary open interval. These formulas also establish a 1-1 correspondence between $\mathscr{R} V_{\kappa}$ and $\mathscr{R} h_{\kappa}=h_{\kappa}^{+}-h_{\kappa}^{+}$.

6. $\kappa$-absolutely continuous and analytic premeasures.

6.1. Definition. A premeasure $\mu \in V_{\kappa}$ is called $\kappa$-absolutely continuous if

$$
\lim _{\tau \rightarrow 0} \operatorname{Var}_{\kappa}\left(\mu-\mu_{\tau}\right)=0
$$

where $\mu_{\tau}$ is the $\tau$-translation of $\mu: \mu_{\tau}((\alpha, \beta))=\mu((\alpha-\tau, \beta-\tau)), \forall(\alpha, \beta) \subset$ T. $L_{\kappa}^{1}$ is the subspace of $V_{\kappa}$ consisting of all $\kappa$-absolutely continuous premeasures.

THEOREM. (a) $L_{\kappa}^{1}$ is a separable Banach space. It consists of those premeasures $\mu \in V_{\kappa}$ that can be approximated in $V_{\kappa}$ by $L^{1}$-measures.

(b) A premeasure $\mu \in V_{\kappa}$ is $\kappa$-absolutely continuous if and only if for every $\varepsilon>0$ there is $a \delta=\delta_{\varepsilon}>0$ such that for any finite system $\left\{I_{j}\right\}_{1}^{n}$ of disjoint intervals $I_{j} \in \mathscr{I}$ such that $\sum_{1}^{n}\left|I_{j}\right|<\delta$, the following inequality holds:

$$
\sum_{1}^{n}\left|\mu\left(I_{j}\right)\right| \leqslant \varepsilon \kappa(E)
$$

where $E$ is the set of endpoints of the $I_{j}$ 's.

Proof. (a) Clearly, $L^{1}$-measures are $\kappa$-absolutely continuous and therefore belong to $L_{\kappa}^{1}$. Suppose that $\mu \in L_{\kappa}^{1}$, i.e. $\mu \in V_{\kappa}$ and satisfies (6.1.1). Consider the Poisson integral $u(z)$ of $\mu$ and the measures $\mu_{r}^{(r)}=u\left(r e^{i t}\right) d t(0<r<1)$, where $d t$ is the normalized Lebesgue measure. We have

$$
\operatorname{Var}_{\kappa}\left(\mu-\mu^{(r)}\right) \leqslant \int \frac{1-r^{2}}{\left|1-r e^{i t}\right|^{2}} \operatorname{Var}_{\kappa}\left(\mu-\mu_{t}\right) d t
$$

Using (6.1.1), as well as the fact that the $w^{*}$-limit of the Poisson kernel (as $r \rightarrow 1^{-}$) is equal to the Dirac's $\delta$-function, we obtain

$$
\lim _{r \rightarrow 1^{-}} \operatorname{Var}_{\kappa}\left(\mu-\mu^{(r)}\right)=0
$$

which proves that $\operatorname{clos} L^{1}=L_{\kappa}^{1}$. 
(b) Suppose that (6.1.2) fails. Then there are systems of intervals $\left\{I_{j}^{(k)}\right\}_{j=1}^{n_{k}}$ $(k=1,2, \ldots)$ such that $\sum_{j=1}^{n_{k}}\left|I_{j}^{(k)}\right| \leqslant 1 / k$, but $\sum_{j=1}^{n_{k}}\left|\mu\left(I_{j}^{(k)}\right)\right| \geqslant a \kappa\left(E^{(k)}\right)$, where $a>0$ and $E^{(k)}$ is the set of the endpoints of $I_{j}^{(k)}\left(j=1,2, \ldots, n_{k}\right)$. For an arbitrary $L^{1}$-measure $\mu_{0}$

$$
\lim _{k \rightarrow \infty} \sum_{j=1}^{n_{k}}\left|\mu_{0}\left(I_{j}^{(k)}\right)\right|=0 .
$$

Since the $\kappa$-entropy of a nonempty set is always $\geqslant 1$, we get from the above that, for sufficiently large $k$,

$$
\sum_{j=1}^{n_{k}}\left|\left(\mu-\mu_{0}\right)\left(I_{j}^{(k)}\right)\right| \geqslant \frac{a}{2} \kappa\left(E^{(k)}\right),
$$

which shows that $\mu \notin L_{\kappa}^{1}$. Thus, (6.1.2) is necessary for $\mu$ to be $\kappa$-absolutely continuous.

If (6.1.2) holds, then the existence of the approximating $L^{1}$-measures is established by a slight adaptation of the method used in [3] for proving a closely related result about "weak" approximation of premeasures of bounded Shannon entropy.

COROllaRY. A $\kappa$-absolutely continuous premeasure vanishes on $\kappa$-Carleson sets.

Proof. Let $\mu \in V_{\kappa}$ and let $E$ be a $\kappa$-Carleson set with complementary intervals $I_{\nu}$. Put

$$
m_{\nu}=\sup \left\{|\mu([\alpha, \beta])|:[\alpha, \beta] \subset I_{\nu}\right\} .
$$

It is easily seen that $\sum m_{\nu}<\infty$. Let $E_{\delta}(\delta>0)$ be the $\delta$-neighborhood of $E$; its complement $\mathbf{T} \backslash E_{\delta}$ is the union of a finite number of closed disjoined intervals $\left[\alpha_{\nu}, \beta_{\nu}\right]$, and we can enumerate them so that $\left[\alpha_{\nu}, \beta_{\nu}\right] \subset I_{\nu}$. We have $\mu\left(E_{\delta}\right)=$ $-\sum_{\nu} \mu\left(\left[\alpha_{\nu}, \beta_{\nu}\right]\right)$; moreover, $\left|\mu\left(\left[\alpha_{\nu}, \beta_{\nu}\right]\right)\right| \leqslant m_{\nu}$ for all $\nu$ and $\delta>0$. Therefore

$$
\lim _{\delta \rightarrow 0} \mu\left(E_{\delta}\right)=-\sum_{\nu} \mu\left(I_{\nu}\right)=\mu(E) .
$$

If $\mu$ is $\kappa$-absolutely continuous, then by (6.1.2)

$$
\left|\mu\left(E_{\delta}\right)\right| \leqslant \kappa\left(\partial E_{\delta}\right) o(1)=o(1) \quad(\delta \rightarrow 0)
$$

and therefore $\mu(E)=\lim _{\delta \rightarrow 0} \mu\left(E_{\delta}\right)=0$.

REMARK. There are premeasures $\mu \in V_{\kappa}$ that vanish on all $\kappa$-Carleson sets without being absolutely continuous. This contrasts with the classical theory, where a Borel measure $\mu$ is absolutely continuous if and only if $\mu(E)=0$ for all $E$ of Lebesgue measure 0 .

6.2. A premeasure $\mu \in V_{\kappa}$ is called analytic if

$$
\hat{\mu}(n)=\int e^{-i n t} d \mu=0 \quad(n=-1,-2, \ldots)
$$

(of course, $\hat{\mu}(0)=0$ for every premeasure $\mu$ ). Analytic premeasures $\mu \in V_{\kappa}$ form a subspace of $V_{\kappa}$ that will be denoted by $H_{\kappa}^{1}$.

A celebrated theorem of the Riesz brothers states that an analytic Borel measure $\mu \in V$ is absolutely continuous, in other words that $H^{1} \subset L^{1}$. There are some 
indications that a similar result might hold for analytic premeasures of bounded $\kappa$-variation; for example, it can be proved that every analytic premeasure of bounded $\kappa$-variation vanishes on all $\kappa$-Carleson sets. ${ }^{5}$

CONJECTURE. $H_{\kappa}^{1} \subset L_{\kappa}^{1}$.

\section{7. $L_{\kappa}^{\infty}$ as the dual of $L_{\kappa}^{1}$.}

7.1. To obtain a description of linear functionals on $L_{\kappa}^{1}$, an extension is needed of the $\kappa$-integral $\int x d \mu$ to the case $x \in L_{\kappa}^{\infty}, \mu=L_{\kappa}^{1}$. Formula (4.2.1), which served as the definition of a $\kappa$-integral for the case $x \in \mathscr{R} C_{\kappa}, \mu \in V_{\kappa}$, still applies, but it is not immediately clear that this extension preserves the bilinearity of $\int x d \mu$. To prove that point observe first that if $x \in \mathscr{R} L_{\kappa}^{\infty}, d \mu \in L^{1}$ (i.e. $\mu$ is absolutely continuous), then the $\kappa$-integral coincides with the Lebesgue integral:

$$
\int x d \mu=\int x(t) \tilde{\mu}^{\prime}(t) d t
$$

In fact, using (2.2.1) we get

$$
\begin{aligned}
\int x(t) \tilde{\mu}^{\prime}(t) d t & =\int\left(y_{0}+\int_{y_{0}}^{\infty} \chi_{y}(t) d y\right) \tilde{\mu}^{\prime}(t) d t \\
& =\int_{y_{0}}^{\infty}\left(\int \chi_{y}(t) \tilde{\mu}^{\prime}(t) d t\right) d y=\int_{y_{0}}^{\infty} \mu\left(F_{y}[x]\right) d y=\int x d \mu .
\end{aligned}
$$

Thus, $\kappa$-integral is bilinear in $x \in L_{\kappa}^{\infty}, d \mu \in L^{1}$. To extend this result to $\mu \in L_{\kappa}^{1}$, we use the following

LEMMA. Let $\left\{\mu_{\kappa}\right\}_{1}^{\infty}$ be a sequence of $L^{1}$-measures converging in $L_{\kappa}^{1}$ to a premeasure $\mu$ :

$$
\lim _{n \rightarrow \infty} \operatorname{Var}_{\kappa}\left(\mu-\mu_{n}\right)=0
$$

Then

$$
\lim _{n \rightarrow \infty} \int x d \mu_{n}=\int x d \mu
$$

for all $x \in L_{\kappa}^{\infty}$.

Proof. We can assume that $x \in \mathscr{R} L_{\kappa}^{\infty}$. Then

$$
\begin{aligned}
\left|\int x d \mu-\int x d \mu_{n}\right| & =\left|\int_{-\infty}^{\infty}\left(\mu-\mu_{n}\right)\left(F_{y}[x]\right) d y\right| \\
& \leqslant \frac{1}{2} \int_{-\infty}^{\infty} \operatorname{Var}_{\kappa}\left(\mu-\mu_{n}\right) \kappa\left(\partial F_{y}[x]\right) d y \\
& =\frac{1}{2} \operatorname{Var}_{\kappa}\left(\mu-\mu_{n}\right)\|x\|_{\kappa} \rightarrow 0 \quad(\kappa \rightarrow \infty)
\end{aligned}
$$

The above Lemma, in combination with the fact that $L^{1}$ is dense in $L_{\kappa}^{1}$, implies that $\int x d \mu$ is linear in $\mu \in L_{\kappa}^{1}$ for every fixed $x \in L_{\kappa}^{\infty}$ and linear in $x \in L_{\kappa}^{\infty}$ for every fixed $\mu \in L_{\kappa}^{1}$.

\footnotetext{
${ }^{5}$ Since this result is inconclusive, its proof is not given here.
} 
The estimates below follow immediately from (4.2.1) and (3.7.1):

$$
\begin{aligned}
& \left|\int x d \mu\right| \leqslant \frac{1}{2}\|x\|_{\kappa} \operatorname{Var}_{\kappa} \mu \quad\left(\forall x \in \mathscr{R} L_{\kappa}^{\infty}, \mu \in L_{\kappa}^{1}\right) ; \\
& \left|\int x d \mu\right| \leqslant \frac{\sqrt{2}}{2}\|x\|_{\kappa} \operatorname{Var}_{\kappa} \mu \quad\left(\forall x \in L_{\kappa}^{\infty}, \mu \in L_{\kappa}^{1}\right) .
\end{aligned}
$$

7.2. TheOREM. (a) Every (real) linear functional $f$ on $\mathscr{R} L_{\kappa}^{1}$ has the form of a $\kappa$-integral

$$
f(\mu)=\int f d \mu
$$

where $f \in \mathscr{R} L_{\kappa}^{\infty}$. Conversely, every function $f \in \mathscr{R} L_{\kappa}^{\infty}$ determines by (7.2.1) a linear functional $f(\mu)$ on $\mathscr{R} L_{\kappa}^{1}$ with the norm

$$
\|f\|^{\mathscr{R}}=\frac{1}{2}\|f\|_{\kappa} .
$$

(b) Every linear functional $f$ on $L_{\kappa}^{1}$ has the form (7.2.1), where $f \in L_{\kappa}^{\infty}$. Conversely, every function $f \in L_{\kappa}^{\infty}$ determines by (7.2.1) a linear functional on $L_{\kappa}^{1}$, and its norm satisfies

$$
\frac{1}{2 \sqrt{2}}\|f\|_{\kappa} \leqslant\|f\| \leqslant \frac{\sqrt{2}}{2}\|f\|_{\kappa} .
$$

Proof. (a) Since the norm of $L_{\kappa}^{1}$ does not exceed that of $L^{1}$, every linear functional $f$ on $\mathscr{R} L_{\kappa}^{1}$ is also a (bounded) linear functional on $\mathscr{R} L^{1}$ and therefore has the form (7.2.1) with some $f(t) \in \mathscr{R} L^{\infty}$; at this point, however, (7.2.1) is valid only for $d \mu \in L^{1}$. We want to show that $f(t) \in \mathscr{R} L_{\kappa}^{\infty}$.

Construct the "smoothed-out" function $f^{h}$ :

$$
f^{h}(t)=\frac{1}{2 h} \int_{-h}^{h} f_{\tau}(t) d \tau=\frac{1}{2 h} \int_{t-h}^{t+h} f(\tau) d \tau \quad(h>0),
$$

and similarly the "smoothed-out" premeasure $\mu^{h}$ :

$$
\mu^{h}=\frac{1}{2 h} \int_{-h}^{h} \mu_{\tau} d \tau
$$

where $\mu_{\tau}$ is the $\tau$-translation of $\mu: \mu_{\tau}(I)=\mu\left(I_{-\tau}\right)$ for all intervals $I, I_{-\tau}=\{t$ : $t+\tau \in I\}$.

For any $f(t) \in L^{\infty}$ and $\mu \in L_{\kappa}^{1}, f^{h}(t)$ is an absolutely continuous function and $d \mu^{h}$ an absolutely continuous measure; therefore the integrals $\int f^{h} d \mu, \int f d \mu^{h}$ exist, the first as a $\kappa$-integral and the second as a Lebesgue integral. We claim that

$$
\int f^{h} d \mu=\int f d \mu^{h} \quad(h>0) .
$$

In fact, fixing $h$ we have for an arbitrary $\varepsilon>0$

$$
\int f^{h} d \mu^{\varepsilon}=\int f d\left(\mu^{\varepsilon}\right)^{h}=\int f d\left(\mu^{h}\right)^{\varepsilon} .
$$

As $\varepsilon \rightarrow 0, \mu^{\varepsilon} \rightarrow \mu$ in $L_{\kappa}^{1}$ and $d\left(\mu^{h}\right)^{\varepsilon} \rightarrow d \mu^{h}$ in $L^{1}$. Taking the limits in (7.2.5) we obtain (7.2.4). 
Now we can represent our functional $f(\mu)\left(\mu \in \mathscr{R} L_{\kappa}^{1}\right)$ as the $w^{*}$-limit of "smooth" functionals $f^{h}(\mu)$ :

$$
f(\mu)=\lim _{h \rightarrow 0} f\left(\mu^{h}\right)=\lim _{h \rightarrow 0} \int f d \mu^{h}=\lim _{h \rightarrow 0} \int f^{h} d \mu=\lim _{h \rightarrow 0} f^{h}(\mu) .
$$

Now, $f^{h}(t) \in \mathscr{R} C_{\kappa}$, and the (real) dual of $\mathscr{R} C_{\kappa}$ is $\mathscr{R} V_{\kappa}$. Therefore the norm of the functional $f^{h}$ on $\mathscr{R} V_{\kappa}$ is $\frac{1}{2}\left\|f^{h}\right\|_{\kappa}$; this is also the norm of the functional $f^{h}$ on $\mathscr{R} L_{\kappa}^{1}$, since $L_{\kappa}^{1}$ is dense in $V_{\kappa}$ in the $w^{*}$-topology of $V_{\kappa}$ considered as the dual of $C_{\kappa}$. By the uniform boundedness theorem, (7.2.6) implies that $\sup _{0<h<1 / 2}\left\|f^{h}\right\|_{\kappa}<\infty$. On the other hand, using the general properties of the $\kappa$-norm (see $\S 3$ ), it is easy to show that

$$
\|f\|_{\kappa}=\lim _{h \rightarrow 0}\left\|f^{h}\right\|_{\kappa} .
$$

Thus $\|f\|_{\kappa}<\infty$ and, by (7.1.2) and (7.2.6),

$$
f(\mu)=\int f d \mu \quad\left(\forall \mu \in \mathscr{R} L_{\kappa}^{1}\right) .
$$

To prove (7.2.2) observe that the functional $f$ on $\mathscr{R} L_{\kappa}^{1}$ is the $w^{*}$-limit of $f^{h}$ as $h \rightarrow 0$, and so, by the general properties of the $w^{*}$-limits,

$$
\|f\|^{\mathscr{R}} \leqslant \lim _{h \rightarrow 0}\left\|f^{h}\right\|^{\mathscr{R}}=\frac{1}{2} \lim _{h \rightarrow 0}\left\|f^{h}\right\|_{\kappa}=\frac{1}{2}\|f\|_{\kappa} .
$$

On the other hand, since $f^{h}$ is a convex combination of $f_{\tau}(-h<\tau<h)$, we have

$$
\frac{1}{2}\left\|f^{h}\right\|_{\kappa}=\left\|f^{h}\right\|^{\mathscr{R}} \leqslant\|f\|^{\mathscr{R}}, \quad \frac{1}{2} \lim _{h \rightarrow 0}\left\|f^{h}\right\|_{\kappa}=\frac{1}{2}\|f\|_{\kappa} \leqslant\|f\|^{\mathscr{R}}
$$

(see (7.1.3)). Thus $\|f\|^{\mathscr{R}}=\frac{1}{2}\|f\|_{\kappa}$.

(b) follows from (7.2.2), (7.1.3) and (3.7.1).

8. $C_{\kappa}$ and $L_{\kappa}^{\infty}$ as Banach algebras.

8.1. Our next object is to show that with a slight adjustment of the $\kappa$-norm the spaces $C_{\kappa}$ and $L_{\kappa}^{\infty}$ become commutative Banach algebras with pointwise multiplication.

Definition. Let $x(t) \in L^{\infty}$. We define the modified $\kappa$-norm as follows:

$$
\|\| x\|\|_{\kappa}=\|x\|_{\infty}+\|x\|_{\kappa}
$$

where $\|x\|_{\infty}=\operatorname{ess} \sup \{|x(t)|: t \in \mathbf{T}\} . \tilde{L}_{\kappa}^{\infty}$ will denote the Banach space of functions $x(t)$ with the norm $\|x\|_{\kappa}<\infty ; \tilde{C}_{\kappa}$ will denote the subspace of $\tilde{L}_{\kappa}^{\infty}$ consisting of continuous functions $x(t) ; \mathscr{R} \tilde{L}_{\kappa}^{\infty} \subset \tilde{L}_{\kappa}^{\infty}$ and $\mathscr{R} \tilde{C}_{\kappa} \subset \tilde{C}_{\kappa}$ consist of real functions.

LEMMA. (a) If $x, y \in \mathscr{R} L_{\kappa}^{\infty}$ and $x(t) \geqslant 0, y(t) \geqslant 0$, then

$$
\|x y\|_{\kappa} \leqslant\|x\|_{\infty}\|y\|_{\kappa}+\|x\|_{\kappa}\|y\|_{\infty} .
$$

(b) If $x, y \in \mathscr{R} \tilde{L}_{\kappa}^{\infty}$, then

$$
\|x y\|_{\kappa} \leqslant 2\left(\|x\|_{\infty}\|y\|_{\kappa}+\|x\|_{\kappa}\|y\|_{\infty}\right)
$$

(c) If $x, y \in \tilde{L}_{\kappa}^{\infty}$, then

$$
\|x y\|_{\kappa} \leqslant 4\left(\|x\|_{\infty}\|y\|_{\kappa}+\|x\|_{\kappa}\|y\|_{\infty}\right) .
$$


Proof. (a) Put $\|x\|=M_{1}$ and $\|y\|=M_{2}$. Then

$$
x(t)=\int_{0}^{M_{1}} \chi_{1}(t, u) d u, \quad y(t)=\int_{0}^{M_{2}} \chi_{2}(t, u) d u,
$$

where $\chi_{1}(t, u)=\chi\left(t ; F_{u}[x]\right)$ and $\chi_{2}(t, u)=\chi\left(t ; F_{u}[y]\right)$ are the characteristic functions of the Lebesgue sets $F_{u}[x]$ and $F_{u}[y]$. Therefore

$$
x(t) y(t)=\int_{0}^{M_{1}} \int_{0}^{M_{2}} \chi_{1}(t, u) \chi_{2}(t, v) d u d v
$$

and

$$
\begin{aligned}
\|x y\|_{\kappa} & \leqslant \int_{0}^{M_{1}} \int_{0}^{M_{2}}\left\|\chi_{1}(\cdot, u) \chi_{2}(\cdot, v)\right\|_{\kappa} d u d v \\
& =\int_{0}^{M_{1}} \int_{0}^{M_{2}} \kappa\left(\partial\left(F_{u}[x] \cap F_{v}[y]\right)\right) d u d v \\
& \leqslant \int_{0}^{M_{1}} \int_{0}^{M_{2}}\left(\kappa\left(\partial F_{u}[x]\right)+\kappa\left(\partial F_{v}[y]\right)\right) d u d v=M_{2}\|x\|_{\kappa}+M_{1}\|y\|_{\kappa} .
\end{aligned}
$$

(b)

$$
\begin{aligned}
\|x y\|_{\kappa}= & \left\|\left(x^{+}-x^{-}\right)\left(y^{+}-y^{-}\right)\right\|_{\kappa} \\
\leqslant & \left(\left\|x^{+}\right\|_{\infty}+\left\|x^{-}\right\|_{\infty}\right)\left(\left\|y^{+}\right\|_{\kappa}+\left\|y^{-}\right\|_{\kappa}\right) \\
& +\left(\left\|x^{+}\right\|_{\kappa}+\left\|x^{-}\right\|_{\kappa}\right)\left(\left\|y^{+}\right\|_{\infty}+\left\|y^{-}\right\|_{\infty}\right) \\
\leqslant & 2\left(\|x\|_{\infty}\|y\|_{\kappa}+\|x\|_{\kappa}\|y\|_{\infty}\right),
\end{aligned}
$$

since $\left\|x^{+}\right\|_{\kappa}+\left\|x^{-}\right\|_{\kappa}=\|x\|_{\kappa},\left\|y^{+}\right\|_{\kappa}+\left\|y^{-}\right\|_{\kappa}=\|y\|_{\kappa}$

(c) Let $x=x_{1}+i x_{2} \in \tilde{L}_{\kappa}^{\infty}$ and $y=y_{1}+i y_{2} \in \tilde{L}_{\kappa}^{\infty}$. Using (8.1.3) and (3.7.1) we find that

$$
\begin{aligned}
\|x y\|_{\kappa} & \leqslant\left\|x_{1} y_{1}-x_{2} y_{2}\right\|_{\kappa}+\left\|x_{1} y_{2}+x_{2} y_{1}\right\|_{\kappa} \\
& \leqslant 2\left[\left(\left\|x_{1}\right\|_{\infty}+\left\|x_{2}\right\|_{\infty}\right)\left(\left\|y_{1}\right\|_{\kappa}+\left\|y_{2}\right\|_{\kappa}\right)+\left(\left\|x_{1}\right\|_{\kappa}+\left\|x_{2}\right\|_{\kappa}\right)\left(\left\|y_{1}\right\|_{\infty}+\left\|y_{2}\right\|_{\infty}\right)\right] \\
& \leqslant 4\left(\|x\|_{\infty}\|y\|_{\kappa}+\|x\|_{\kappa}\|y\|_{\infty}\right) .
\end{aligned}
$$

THEOREM. If $x, y \in \tilde{L}_{\kappa}^{\infty}$, then $x y \in \tilde{L}_{\kappa}^{\infty}$ and

$$
\||x y|\|_{\kappa} \leqslant 4\left|\| x \| \left\|_ { \kappa } \left|\|y \mid\|_{\kappa} .\right.\right.\right.
$$

Thus $\tilde{L}_{\kappa}^{\infty}$ and $\tilde{C}_{\kappa}$ are Banach algebras.

Proof. By the preceding Lemma,

$$
\begin{aligned}
\|x y\|_{\kappa} & =\|x y\|_{\infty}+\|x y\|_{\kappa} \leqslant\|x\|_{\infty}\|y\|_{\infty}+4\left(\|x\|_{\infty}\|y\|_{\kappa}+\|x\|\left\|_{\kappa}\right\| y \|_{\infty}\right) \\
& \leqslant 4\left(\|x\|_{\infty}+\|x\|_{\kappa}\right)\left(\|y\|_{\infty}+\|y\|_{\kappa}\right)=4\|x\|\left\|_{\kappa}\right\| y\|\|_{\kappa} . \quad \text { Q.E.D. }
\end{aligned}
$$

8.2. The following theorem shows that the structure of maximal ideals of the Banach algebra $\tilde{C}_{k}$ is identical with that of the algebra $C$ of all continuous functions.

THEOREM. Every point $t_{0}=\mathbf{T}$ determines a maximal ideal $M_{t_{0}}=\left\{x \in \tilde{C}_{\kappa}: x\left(t_{0}\right)=\right.$ 0 ) of $\tilde{C}_{\kappa}$, and vice versa.

Proof. An equivalent formulation is that every nonzero linear multiplicative functional $F(x)$ on $\tilde{C}_{\kappa}$ has the form $F_{t_{0}}(x)=x\left(t_{0}\right)\left(t_{0} \in \mathbf{T}\right) . \tilde{C}_{\kappa}$ is generated by two 
elements $g_{1}=e^{i t}$ and $g_{2}=e^{-i t}$. Let $F\left(g_{1}\right)=\zeta$ and therefore $F\left(g_{2}\right)=\zeta^{-1}$, where $0 \neq \zeta \in \mathbf{C}$. Using the definition of the $\kappa$-norm we find that $\left\|e^{i n t}\right\|_{\kappa}=O(|n| \kappa(1 /|n|))$ $(|n| \rightarrow \infty)$. On the other hand, $F\left(g_{1}^{n}\right)=\zeta^{n}, F\left(g_{2}^{n}\right)=\zeta^{-n}(n=0,1,2, \ldots)$. Since the functional $F$ is bounded, this is possible only if $|\zeta|=1$; let $\zeta=e^{i t_{0}}\left(t_{0} \in \mathbf{T}\right)$. We have $F(P)=P\left(t_{0}\right)$ for all trigonometric polynomials $P$, and, since polynomials are dense in $\tilde{C}_{\kappa}, F(x)=x\left(t_{0}\right)$ for all $x \in \tilde{C}_{\kappa}$.

8.3. The analytic subalgebra $\tilde{A}_{\kappa}$ of $\tilde{C}_{k}$ is contained in the disk algebra $A$ and contains the algebra $A^{1}=\left\{f: f^{\prime} \in A\right\}$. Similarly, $\tilde{H}_{\kappa}^{\infty}$ (the analytic subalgebra of $\left.\tilde{L}_{\kappa}^{\infty}\right)$ lies between $H^{\infty}$ and $H_{1}^{\infty}=\left\{f: f^{\prime} \in H^{\infty}\right\}$. A systematic study of $\tilde{A}_{\kappa}$ and $\tilde{H}_{\kappa}^{\infty}$ may reveal some new properties of classical spaces of analytic functions. Such a study, however, is beyond the scope of this paper.

\section{A generalization of two classical convergence tests for Fourier series.}

9.1. The Dirichlet-Jordan (D-J) convergence test for Fourier series [1] states that the (symmetrical) partial sums $S_{n}(t ; f)$ of the Fourier series $S[f]$ of a function $f(t)$ $(t \in \mathbf{T})$ of bounded variation tend to $\frac{1}{2}[f(t+0)+f(t-0)]$ as $n \rightarrow \infty$; if $f(t)$ is also continuous, then $S_{n}(t) \rightarrow f(t)$ uniformly.

The Dini-Lipschitz (D-L) test [1] states that $S_{n}(t ; f) \rightarrow f(t)$ uniformly if the modulus of continuity $\omega(\delta)$ of $f(t)$ is $o\left(|\log \delta|^{-1}\right)(\delta \rightarrow 0)$.

The proof of the D-J test is based on the classical $C-V$ duality. However, if instead of $C-V$ duality we use the Dini-entropy-norm-Dini-variation duality $\left(C_{d}-V_{d}\right)$, we obtain a new test that includes both the D-J and the D-L tests.

Since we need here only one special case of $\kappa$-norm and $\kappa$-variation, namely $\kappa(s)=\left(1+\frac{1}{2}|\log s|\right)^{-1}$, we introduce special notations for this case: $\|\cdot\|_{\kappa}=\|\cdot\|_{d}$, $\operatorname{Var}_{\kappa} f=\operatorname{Var}_{d} f, C_{\kappa}=C_{d}, V_{\kappa}=V_{d}$. It is also expedient to apply the symbol $\operatorname{Var}_{d} f$ to functions $f(t)$ rather than to premeasures, so that $\operatorname{Var}_{d} f$ really means

$$
\operatorname{Var}_{d} f(t)=\sup \frac{\sum_{j=1}^{n+1}\left|f\left(t_{j}\right)-f\left(t_{j-1}\right)\right|}{\sum_{j=1}^{n+1} \kappa\left(\left(t_{j}-t_{j-1}\right) / 2 \pi\right)},
$$

where $\kappa(s)=\left(1+\frac{1}{2}|\log s|\right)^{-1}$, and the supremum is taken over all $t_{0}<t_{1}<\cdots<$ $t_{n+1}=t_{0}+2 \pi(n=1,2, \ldots)$. We shall also assume that functions $f \in V_{d}$ are normalized so that

$$
f(t)=\frac{1}{2}(f(t+0)+f(t-0)) \quad(\forall t \in \mathbf{T}) .
$$

9.2. We start with the following formula [1] for the partial sums $S_{n}(t ; f)$ of the Fourier series $S[f]$ :

$$
S_{n}(t ; f)=f(t)+\int \Phi_{t}(\tau) D_{n}(\tau) d \tau
$$

where

$$
\begin{gathered}
\Phi_{t}(\tau)=\Phi_{t}(\tau ; f)=\frac{1}{2}[f(t+\tau)+f(t-\tau)]-f(t), \\
D_{n}(\tau)=\sin (n+1 / 2) \tau / \sin (\tau / 2)
\end{gathered}
$$


and $d \tau$ is the normalized Lebesgue measure on $\mathbf{T}$. We introduce now the "integrated Dirichlet kernel"' $\mathscr{E}_{n}(t)$ :

$$
\mathscr{E}_{n}(t)=\left\{\begin{array}{l}
\int_{t}^{\pi} D_{n}(\tau) d \tau=\frac{\pi-t}{2 \pi}-\frac{1}{\pi} \sum_{k=1}^{n} \frac{\operatorname{Sin} k t}{k} \quad(0<t \leqslant \pi), \\
-\mathscr{E}_{n}(-t) \quad(-\pi \leqslant t<0), \\
0 \quad(t=0) .
\end{array}\right.
$$

Clearly,

$$
\frac{d}{d t} \mathscr{E}_{n}(t)=-D_{n}(t)+\delta_{0}(t)
$$

where $\delta_{0}(t)$ is the unit Dirac mass at $t=0$. Since $\Phi_{t}(0)=0$, we can write (9.2.1) as a Stieltjes integral

$$
S_{n}(t ; f)=f(t)-\int \Phi_{t}(\tau) d \mathscr{E}_{n}(\tau)
$$

Integrating by parts we obtain

$$
S_{n}(t ; f)=f(t)+\int \mathscr{E}_{n}(\tau) d \Phi_{t}(\tau)
$$

We need now the following

LEMMA.

$$
\left\|\mathscr{E}_{n}\right\|_{d} \leqslant 7 \quad(n=1,2, \ldots) .
$$

Proof. A simple computation shows that $\mathscr{E}_{n}(t)$ satisfies

$$
\left|\mathscr{E}_{n}(t)\right| \leqslant \min \left\{\frac{1}{2}, \frac{2}{(2 n+1)|t|}\right\} \quad(-\pi \leqslant t \leqslant \pi) .
$$

$\mathscr{E}_{n}(t)$ is monotone in each of the intervals $(2 k \pi /(2 n+1), 2(k+1) \pi /(2 n+1))$ $(k=0,1, \ldots, n-1)$ and $(2 n \pi /(2 n+1), \pi)$. Therefore the level set $E_{y}\left[\mathscr{E}_{n}\right]\left(-\frac{1}{2}<\right.$ $\left.y<\frac{1}{2}\right)$ lies in the interval $[-2 /(2 n+1)|y|, 2 /(2 n+1)|y|]$ and has at most $2 / \pi|y|$ +1 points if $|y|>1 / n \pi ; E_{y}\left[\mathscr{E}_{n}\right]$ has at most $2 n+3$ points if $|y| \leqslant 1 / n \pi$. This easily leads to the following estimate for the Dini-entropy of $E_{y}\left[\mathscr{E}_{n}\right]$ :

$$
\begin{array}{ll}
\kappa_{d}\left(E_{y}\left[\mathscr{E}_{n}\right]\right) \leqslant(2 n+3)\left(1+\frac{1}{2} \log (2 n+3)\right)^{-1} \quad\left(|y| \leqslant \frac{1}{n \pi}\right), \\
\kappa_{d}\left(E_{y}\left[\mathscr{E}_{n}\right]\right) \leqslant 1+\frac{2}{\pi|y|}\left(1+\frac{1}{2} \log (2 n+1)\right)^{-1} \quad\left(|y|>\frac{1}{n \pi}\right) .
\end{array}
$$

Thus

$$
\begin{aligned}
\left\|\mathscr{E}_{n}\right\|_{d} & =\int_{-1 / 2}^{1 / 2} \kappa_{d}\left(E_{y}\left[\mathscr{E}_{n}\right]\right) d y \leqslant \frac{2(2 n+3)}{n \pi}+1+\frac{8(\log n \pi-\log 2)}{\pi(2+\log (2 n+1))} \\
& <\frac{10}{\pi}+1+\frac{8}{\pi}<7 . \quad \text { Q.E.D. }
\end{aligned}
$$


Using (9.2.4), (9.2.5), (4.2.3) and observing that $\operatorname{Var}_{d} \Phi_{\tau} \leqslant \operatorname{Var}_{d} f$, we arrive at the following result.

THEOREM.

$$
\max _{t \in \mathbf{T}}\left|S_{n}(t ; f)\right| \leqslant\|f\|_{\infty}+\frac{7}{2} \operatorname{Var}_{d} f .
$$

9.3. Having established the boundedness of $S_{n}(t ; f)$ for $f \in V_{\kappa}$, we turn now to the problem of convergence. First observe that the argument used in proving (9.2.5) also yields the following

LEMMA. Let $\mathscr{E}_{n, \delta}(t)=\mathscr{E}_{n}(t)\left(1-\chi_{\delta}(t)\right)$, where $\chi_{\delta}(t)$ is the characteristic function of the interval $(-\delta, \delta)(0<\delta<\pi)$. Then

$$
\lim _{n \rightarrow \infty}\left\|\mathscr{E}_{n, \delta}\right\|_{d}=0
$$

From (9.2.4) we obtain $(\delta>0$ fixed):

$$
\begin{aligned}
S_{n}(t ; f)-f(t)= & \int \mathscr{E}_{n, \delta}(\tau) d \Phi_{t}(\tau)+\int \mathscr{E}_{n}(\tau) \chi_{\delta}(\tau) d \Phi_{t}(\tau) \\
= & \int \mathscr{E}_{n, \delta}(\tau) d \Phi_{t}(\tau)+\int \mathscr{E}_{n}(\tau) d\left(\Phi_{t}(\tau) \chi_{\delta}(\tau)\right) \\
& -\mathscr{E}_{n}(-\delta) \Phi_{t}(-\delta)+\mathscr{E}_{n}(\delta) \Phi_{t}(\delta)
\end{aligned}
$$

Therefore, by (9.3.1) and (9.2.5),

$$
\varlimsup_{n \rightarrow \infty}\left|S_{n}(t ; f)-f(t)\right| \leqslant 7 \operatorname{Var}_{d}\left(\Phi_{t} \chi_{\delta}\right)+\frac{1}{2}\left(\left|\Phi_{t}(-\delta)\right|+\left|\Phi_{t}(\delta)\right|\right) .
$$

The last term tends to 0 as $\delta \rightarrow 0$, and so we obtain

$$
\varlimsup_{n \rightarrow \infty}\left|S_{n}(t ; f-f(t))\right| \leqslant 7 \underset{\delta \rightarrow 0}{\lim _{\delta \rightarrow 0}} \operatorname{Var}_{d}\left(\Phi_{t} \chi_{\delta}\right) .
$$

In order to obtain convergence of $S_{n}(t ; f)$, an additional condition has to be imposed on $f(t)$.

Definition. A function $f(t) \in V_{d}$ is said to be of vanishing $d$-variation at $t_{0}$ if $\operatorname{Var}_{d}\left\{\left(f(t)-f\left(t_{0}\right)\right) \chi_{\delta}(t)\right\} \rightarrow 0$ as $\delta \rightarrow 0$, where $\chi_{\delta}(t)$ is the characteristic function of the interval $\left(t_{0}-\delta, t_{0}+\delta\right)$. If this takes place at every point $t_{0} \in \mathbf{T}, f(t)$ is said to be of vanishing $d$-variation on $\mathbf{T}$.

REMARK 1. For the classical variation, if $f(t)$ is of bounded variation on $\mathbf{T}$ and continuous at $t_{0}$, then $f(t)$ is of vanishing variation at $t_{0}$. However, for the $\kappa$-variation (and, in particular, for the $d$-variation) this is generally not true.

REMARK 2. If $f(t)$ is $d$-absolutely continuous, then $f(t)$ is of vanishing $d$-variation on $\mathbf{T}$. However, the converse is not true.

REMARK 3. If the modulus of continuity $\omega(\delta)$ of $f(t)$ is $O\left(|\log \delta|^{-1}\right)$, then $f(t)$ is of bounded $d$-variation. If $\omega(\delta)=o\left(|\log \delta|^{-1}\right)$, then $f(t)$ is of vanishing $d$-variation, and even $d$-absolutely continuous.

The following result is a direct consequence of (9.3.2) and the above definition. 
THEOREM. If $f(t)$ is of vanishing d-variation at $t_{0}\left(\right.$ or, more generally, if $\Phi_{t_{0}}(\tau)$ is of vanishing $d$-variation at $\tau=0$ ), then

$$
\lim _{n \rightarrow \infty} S_{n}\left(t_{0} ; f\right)=f\left(t_{0}\right) .
$$

If $f(t)$ is of vanishing d-variation on $\mathbf{T}$ (in particular, if $f(t)$ is d-absolutely continuous), then

$$
\lim _{n \rightarrow \infty} S_{n}(t ; f)=f(t)
$$

uniformly on $\mathbf{T}$.

9.4. If $f$ is continuous and of bounded variation (in particular, if $f$ is absolutely continuous), then by the D-J test

$$
\lim _{n \rightarrow \infty}\left\|f-S_{n}(f)\right\|_{\infty}=0 .
$$

However, we saw that the same conclusion still holds under much weaker assumptions. It is natural therefore to expect that the assumptions of the D-L uniform convergence test should imply a stronger conclusion than (9.4.1). This is in fact the case. First we establish a result about boundedness of $d$-norms of the partial sums $S_{n}(f)$.

LEMMA. If $f(t)$ is of bounded variation, then

$$
\left\|f-S_{n}(f)\right\|_{d} \leqslant 7 \operatorname{Var} f, \quad\left\|S_{n}(f)\right\|_{d} \leqslant 8 \operatorname{Var} f
$$

for all $n \geqslant 1$.

Proof. (9.2.4) can be rewritten in the following form:

$$
\begin{aligned}
S_{n}(t ; f)-f(t) & =\frac{1}{2} \int \mathscr{E}_{n}(\tau) d_{\tau}[f(t+\tau)+f(t-\tau)] \\
& =\frac{1}{2} \int\left[\mathscr{E}_{n}(\tau-t)-\mathscr{E}_{n}(t-\tau)\right] d f(\tau)=\int \mathscr{K}_{n}(t, \tau) d f(\tau),
\end{aligned}
$$

where $\mathscr{K}_{n}(t, \tau)=\frac{1}{2}\left[\mathscr{E}_{n}(\tau-t)-\mathscr{E}_{n}(t-\tau)\right]$. We have $\left\|\mathscr{K}_{n}(\cdot, \tau)\right\|_{d} \leqslant 7 \quad(\forall \tau \in \mathbf{T})$; thus

$$
\left\|S_{n}(f)-f\right\|_{d} \leqslant \int\|\mathscr{K}(\cdot, \tau)\|_{d}|d f(\tau)| \leqslant 7 \operatorname{Var} f .
$$

Since $\|f\|_{d} \leqslant \operatorname{Var} f$, we also have

$$
\left\|S_{n}(f)\right\|_{d} \leqslant\|f\|_{d}+\left\|S_{n}(f)-f\right\|_{d} \leqslant 8 \operatorname{Var} f .
$$

Now we can considerably improve (9.4.1). To simplify the formulation, we confine ourselves to the case of an absolutely continuous $f(t)$.

THEOREM. Suppose that $f(t)$ is absolutely continuous on T. Then

$$
\lim _{n \rightarrow \infty}\left\|f-S_{n}(f)\right\|_{d}=0 .
$$


Proof. $f$ can be approximated by a trigonometric polynomial $P$ so that

$$
\operatorname{Var}(f-P)<\varepsilon,
$$

where $\varepsilon>0$ is arbitrary. We have by (9.4.2)

$$
\varlimsup_{n \rightarrow \infty}\left\|f-S_{n}(f)\right\|_{d}=\varlimsup_{n \rightarrow \infty}\left\|(f-P)-S_{n}(f-P)\right\|_{d} \leqslant 7 \varepsilon .
$$

Since $\varepsilon$ is arbitrary this proves (9.4.3).

REMARK. There exist in the literature some related results concerning convergence of Fourier series. A. M. Garsia and S. Sawyer [4] considered continuous functions with logarithmically integrable Banach indicatrix and proved that such functions have uniformly convergent Fourier series. D. Waterman [5] introduced the class of functions of "harmonic bounded variation" that also have uniformly convergent Fourier series. R. A. Fefferman [6] introduced functions of "finite entropy" which include our space $C_{\kappa}$ with $\kappa(s)=s(1+|\log s|)$. It would be interesting to know more about connections between all these classes of functions.

\section{REFERENCES}

1. A. Zygmund, Trigonometric series, Cambridge Univ. Press, Cambridge, 1959.

2. B. Korenblum, An extension of the Nevanlinna theory, Acta Math. 135 (1975), 187-219.

3. A Beurling-type theorem, Acta Math. 138 (1977), 265-293.

4. A. M. Garsia and S. Sawyer, On some classes of continuous functions with convergent Fourier series, J. Math. Mech. 13 (1964), 589-601.

5. D. Waterman, On convergence of Fourier series of functions of generalized bounded variation, Studia Math. 44 (1972), 107-117.

6. R. A. Fefferman, A theory of entropy in Fourier analysis, Adv. in Math. 30 (1978), 171-201.

Department of Mathematics, State University of New York at Albany, Albany, New York 12222 